\title{
RNA sequencing-based exploration of the effects of far-red light on IncRNAs involved in the shade-avoidance response of D. officinale
}

\author{
Hansheng Li ${ }^{1}$, Wei Ye ${ }^{2}$, Yaqian Wang ${ }^{1}$, Xiaohui Chen ${ }^{3}$, Yan Fang ${ }^{1}$, Gang Sun ${ }^{\text {Corresp. } 1}$ \\ ${ }^{1}$ College of Resources and Chemical Engineering, Sanming University, Sanming, China \\ 2 The Institute of Medicinal Plant, Sanming Academy of Agricultural Science, Shaxian, China \\ 3 Institute of Horticultural Biotechnology, Fujian Agriculture and Forestry University, Fuzhou, China \\ Corresponding Author: Gang Sun \\ Email address: sungang@nenu.edu.cn
}

Dendrobium officinale ( $D$. officinale) is a valuable medicinal plant with a low natural survival rate, and its shade-avoidance response to far-red light is as an important strategy used by the plant to improve its production efficiency. However, the IncRNAs that play roles in the shade-avoidance response of $D$. officinale have not yet been investigated. This study found that an appropriate proportion of far-red light can have several effects, including increasing the leaf area and accelerating stem elongation, in $D$. officinale. The effects of different far-red light treatments on $D$. officinale were analysed by RNA sequencing technology, and a total of 69 and 78 IncRNAs were differentially expressed in experimental group 1 (FR1) versus the control group (CK) (FR1-CK) and in experimental group 4 (FR4) versus the CK (FR4-CK), respectively. According to GO and KEGG analyses, most of the differentially expressed IncRNA targets are involved in the membrane, some metabolic pathways, hormone signal transduction, and 0-methyltransferase activity, among other functions. Physiological and biochemical analyses showed that far-red light promoted the accumulation of flavonoids, alkaloids, carotenoids and polysaccharides in $D$. officinale. The effect of far-red light on $D$. officinale might be closely related to the cell membrane and $\mathrm{Ca}^{2+}$ transduction. Based on a Cytoscape analysis and previous research, this study also found that MSTRG.38867.1, MSTRG.69319.1, and MSTRG.66273.1, among other components, might participate in the far-red light signalling network through their targets and thus regulate the shade-avoidance response of $D$. officinale. These findings will provide new insights into the shade-avoidance response of $D$. officinale. 


\section{RNA sequencing-based exploration of the effects of far-red light on IncRNAs}

\section{2 involved in the shade-avoidance response of $D$. officinale}

3 Hansheng Li ${ }^{1}$, Wei Ye ${ }^{2}$, Yaqian Wang ${ }^{1}$, Xiaohui Chen ${ }^{3}$, Yan Fang ${ }^{1}$ and Gang Sun ${ }^{1 *}$

41 College of Resources and Chemical Engineering, Sanming University, Sanming 365004, China;

52 The Institute of Medicinal Plant, Sanming Academy of Agricultural Science, Shaxian 365509, China

63 Institute of Horticultural Biotechnology, Fujian Agriculture and Forestry University, Fuzhou 350007, China

7 Corresponding Author: Gang Sun

8 Email address: sungang@nenu.edu.cn

10 ABSTRACT

11 Dendrobium officinale (D. officinale) is a valuable medicinal plant with a low natural survival rate, and its

12 shade-avoidance response to far-red light is as an important strategy used by the plant to improve its

13 production efficiency. However, the lncRNAs that play roles in the shade-avoidance response of D. officinale

have not yet been investigated. This study found that an appropriate proportion of far-red light can have

several effects, including increasing the leaf area and accelerating stem elongation, in $D$. officinale. The effects

of different far-red light treatments on $D$. officinale were analysed by RNA sequencing technology, and a total

of 69 and 78 lncRNAs were differentially expressed in experimental group 1 (FR1) versus the control group

(CK) (FR1-CK) and in experimental group 4 (FR4) versus the CK (FR4-CK), respectively. According to GO

and KEGG analyses, most of the differentially expressed lncRNA targets are involved in the membrane, some metabolic pathways, hormone signal transduction, and O-methyltransferase activity, among other functions.

Physiological and biochemical analyses showed that far-red light promoted the accumulation of flavonoids, 
22 alkaloids, carotenoids and polysaccharides in $D$. officinale. The effect of far-red light on $D$. officinale might be

23 closely related to the cell membrane and $\mathrm{Ca}^{2+}$ transduction. Based on a Cytoscape analysis and previous research, this study also found that MSTRG.38867.1, MSTRG.69319.1, and MSTRG.66273.1, among other components, might participate in the far-red light signalling network through their targets and thus regulate the shade-avoidance response of $D$. officinale. These findings will provide new insights into the shade-avoidance response of $D$. officinale.

Subjects Agricultural Science, Biotechnology, Plant Science

Keywords Dendrobium officinale Kimura et Migo, Far-red light, Shade-avoidance response, lncRNAs, RNA-

\section{INTRODUCTION}

Dendrobium officinale Kimura et Migo (D. officinale) is a valuable medicinal plant species that can be used to nourish the stomach and strengthen the body's immunity and that exhibits antitumour and antiaging activity (Sun et al., 2017). Modern chemical and pharmacological studies have shown that D. officinale is rich in polysaccharides, alkaloids, phenols and other secondary metabolites (Sun et al., 2017). Researchers have used different methods to increase the yield of the pharmaceutical ingredients of D. officinale. For example, Wang et al. (2016) compared the medicinal value of $D$. officinale under different cultivation practices and found that the content of polysaccharides, flavonoids and polyphenols was the highest under shaded conditions. Qin et al. (2018) determined the polysaccharide content of $D$. officinale during different years of growth, and their results showed that 5-year-old D. officinale plants had the highest polysaccharide content, followed by 3-yearold plants. Dai et al. (2018) showed that plant growth regulators can promote the growth and development of 
D. officinale plants, increase their resistance to oxidative enzymes, and regulate their chlorophyll content to

44

increase their stress resistance and the accumulation of polysaccharides.

During plant growth, various internal and external factors can affect the synthesis of medicinal components, and light regulation is considered one of the important methods for improving the yield of these components. A study of the accumulation of polysaccharides in the original bulbs of D. officinale under different LED illumination conditions revealed that red-blue mixed light exerts the most obvious effect on the accumulation of polysaccharides. The yield of polysaccharides under 1:3 red light:blue light was the most ambiguous (Hou et al., 2013). Xu et al. (2012) reported that the polysaccharide content of D. officinale protocorms was highest (up to $16.88 \%$ ) after $30 \mathrm{~d}$ of exposure to a light intensity of $20001 \mathrm{x}$. However, the optimal alkaloid content (up to $0.028 \%$ ) was detected after $30 \mathrm{~d}$ of exposure to a light intensity of $500 \mathrm{~lx}$.

One of the most important effects of 730-nm far-red light on plants is the shade-avoidance response (Chen et al., 2015). The shade-avoidance response of most plants involves a unique set of morphological changes and characteristics, such as an increase in the leaf area, plant height, and dry matter accumulation and changes in the photosynthetic physiological characteristics (Yang et al., 2017; Schambow et al., 2019). Kasulin et al. (2013) reported that the height of Arabidopsis thaliana (A. thaliana) can increase through hypocotyl and stem elongation so that the plants protrude higher than others in a population. The red light:far-red light (R:FR) ratio in the upper layer of a population is similar to the normal value, and plants in this layer receive relatively more available light energy. The shade-avoidance response of plants can also regulate the growth period of the plant such that the flowering period occurs sooner, the vegetative period is shortened, and reproductive growth is completed more quickly (Kutschera et al., 2013). Therefore, 730-nm far-red light might promote an increase in the leaf area and the elongation of stem segments, among other effects, in D. officinale, and these effects 
would in turn improve the production efficiency.

The understanding of the molecular mechanism through which far-red light affects the plant shadeavoidance response has increased in model plant species. The plant shade-avoidance response is mediated mainly by phytochrome B (PHYB), which regulates the expression of downstream genes by phytochromeinteracting bHLH factors (PIFs) (Amanda et al., 2016). Phytochrome is transported into the nucleus after being converted into an active far-red light-type (Pfr) configuration by light, and Pfr in the nucleus can directly interact with PIFs (Amanda et al., 2016). PIFs are phosphorylated in comparison with photoactivated phytochrome and are then degraded by the $26 \mathrm{~S}$ proteasome. The stability of PIFs plays an important role in the plant shade-avoidance response (Xie et al., 2017). PIF4 and PIF5 mediate stalk elongation in plants. Under a low R:FR ratio, Pfr is converted into the inactive red light-type (Pr) configuration by light and is exported from the nucleus, which enhances the stability of PIF4 and PIF5 and promotes regulation of the expression of genes involved in stem elongation (Lorrain et al., 2008; Shi et al., 2018). In addition, cryptochrome can mediate the shade-avoidance response through the CRY-SPA1/COP1 pathway and inhibit a plant's shade-avoidance response by preventing the degradation of the positive photoregulatory regulators elongated hypocotyl 5 (HY5) and long hypocotyl in far red 1 (HFR1) (Robson et al., 2010; Sellaro et al., 2011). Recent studies have shown that SPA1 is one of three negative regulators of photomorphogenesis and might participate in the plant shadeavoidance response by regulating the accumulation of phytochrome and cryptochrome (Sellaro et al., 2011).

The involvement of long noncoding RNAs (lncRNAs) in the plant shade-avoidance response has not been previously studied. Due to the rapid development of sequencing technology, low-abundance transcripts at the genome-wide transcription level have been detected. lncRNAs, which are noncoding RNAs longer than 200 nucleotides, have extremely complex and important biological functions; they not only regulate gene 
85

86

87

88

89

90

91

92

93

94

95

96

97

98

99

100

101

102

103

104

105 expression at the epigenetic, transcription, and posttranscriptional levels but also participate in the regulation of many various biological processes, such as genomic imprinting, chromosome remodelling, and transcriptional activation (Garima et al., 2011; Wang et al., 2017). Recent studies have shown that lncRNAs play an important role in the plant response to external factors. The treatment of $A$. thaliana plants with drought, cold and high salt stress significantly changed the expression levels of 1832 lncRNAs. The expression of individual lncRNAs was even upregulated by 22-fold, which indicated that lncRNAs are important regulators of plant responses to external factors and thereby help plants better adapt to different environmental conditions (Liu et al., 2012). Wang et al. (2014) identified a large number of lncRNAs that are activated in response to light in $A$. thaliana, and Shuai et al. (2014) reported 504 lncRNAs that are activated in response to drought in Populus euphratica (P. euphratica). Studies on the response of lncRNAs to external factors have been performed in various plant species, such as $A$. thaliana, Oryza sativa (O. sativa), P. euphratica, Triticum aestivum, Zea mays and Lycopersicon esculentum, but the effects of far-red light on lncRNAs have not yet been investigated in $D$. officinale.

In this study, we used high-throughput sequencing technology to identify putative lncRNAs and investigated their expression profiles in Dendrobium under different illumination patterns. The specific lncRNAs identified by comparing and analysing the sequence data from the treatment and control groups were further studied to assess their involvement in the shade-avoidance response of $D$. officinale induced by far-red light. Moreover, the signal transduction pathway of the lncRNAs involved in the shade-avoidance response of D. officinale was determined. These findings provide new insights for the high-yield production of medicinal components of $D$. officinale.

Peer] reviewing PDF | (2020:06:49729:4:0:NEW 17 Dec 2020) 
MATERIALS AND METHODS

107

108

109

110

111

112

113

114

115

116

117

118

119

120

121

122

123

124

125

126

\section{Plant material and light treatments}

Tissue culture-generated seedlings of $D$. officinale were provided by the Sanming Academy of Agricultural

Sciences, and tissue culture-generated seedlings with three to four true leaves, a seedling height of approximately $25 \mathrm{~mm}$, a stem diameter of approximately $1.8 \mathrm{~mm}$ and a leaf area of approximately $30 \mathrm{~mm}^{2}$ were selected for light treatment. The selected tissue culture-generated $D$. officinale seedlings were placed in a growth chamber for $120 \mathrm{~d}$, and 15 bottles were used for each treatment. The light treatments comprised exposure to red light $(660 \mathrm{~nm})$, blue light $(450 \mathrm{~nm})$, and far-red light $(730 \mathrm{~nm})$; in all treatments, the total light intensity was $200 \mu \mathrm{mol} \mathrm{m} \mathrm{m}^{-2} \mathrm{~s}^{-1}$, the photoperiod was $12 \mathrm{~h} \mathrm{~d}^{-1}$, the humidity ranged from $55 \%$ to $60 \%$, and the temperature was $25 \pm 2{ }^{\circ} \mathrm{C}$. In this study, a group treated with a red light intensity:blue light intensity:far-red light intensity ratio of 100:100:0 served as the control group (CK), and the groups treated with red light intensity:blue light intensity:far-red light intensity ratios of 80:80:40 (experimental group 1 (FR1)), 60:60:80 (experimental group 2 (FR2)), 50:50:100 (experimental group 3 (FR3)), 40:40:120 (experimental group 4 (FR4)), and 20:20:160 (experimental group 5 (FR5)) served as the experimental groups. The tissue culturegenerated seedlings of $D$. officinale were maintained on half-strength Murashige and Skoog (MS) media (6 g $\mathrm{L}^{-1}$ agar and $30 \mathrm{~g} \mathrm{~L}^{-1}$ sucrose, $\mathrm{pH}$ 5.8) supplemented with $1 \mathrm{~g} \mathrm{~L}^{-1}$ activated carbon and $50 \mathrm{~g} \mathrm{~L}^{-1}$ mashed banana and subcultured every $120 \mathrm{~d}$. Half-strength Murashige and Skoog (MS) media (6 g L L agar and $30 \mathrm{~g} \mathrm{~L}^{-1}$ sucrose, $\mathrm{pH}$ 5.8) supplemented with $1 \mathrm{~g} \mathrm{~L}^{-1}$ activated carbon was used for the light treatments. The all samples were frozen in liquid nitrogen and stored at $-80{ }^{\circ} \mathrm{C}$ for high-throughput sequencing, nucleic acid extraction, functional metabolite content determination and antioxidant enzyme activity assays, etc.. The FR1 versus CK, FR4 versus CK, and FR4 versus FR1 comparisons are denoted FR1-CK, FR4-CK, and FR4-FR1, respectively. 


\section{IncRNA library construction and Illumina HiSeq sequencing}

128 According to their phenotypic differences, samples of D. officinale after three treatments-CK, FR1 and

129 FR4 - were selected for high-throughput sequencing, and each treatment involved three biological replicates.

130 Total RNA extraction and determination (Fig. S1; Table S1) referred the method described in Li et al. (2019).

131 Ribosomal RNA (rRNA) was eliminated from the purified RNA using the Ribo-Zero rRNA Removal Kit

132 (Epicentre, Madison, WI, USA). Strand-specific cDNA was synthesized to construct nine D. officinale

133 sequencing libraries using the TruSeq ${ }^{\circledR}$ Stranded Kit (Illumina), ribonuclease H, and DNA polymerase I. Raw

134 data in the fastq format were obtained using the Illumina HiSeq sequencing platform, and low-quality reads,

135 reads with a high $\mathrm{N}$ content, and adapter contamination were removed to obtain clean reads. Sequencing

136 library preparation and high-throughput sequencing were subsequently performed using the Illumina HiSeq

137 platform (Beijing, China). All sequencing data of $D$. officinale after the different light treatments were

138 deposited in the National Center for Biotechnology Information (NCBI) Sequence Read Archive (accession

139 number PRJNA638348).

140 The clean reads were aligned to the Dendrobium officinale reference genome (Zhang et al., 2016) using

141 Hierarchical Indexing for Spliced Alignment of Transcripts (HISAT) software for transcript assembly, and

142 transcripts with lengths below 200 bp and lower expression (FPKM $\leq 0.5)$ were filtered out. The reference

143 genome version of Dendrobium officinale in this manuscript was updated on April 11, 2019. This new version

144 could be found on the website (ftp//ftp.ncbinlm.nih.gov/genomes/all/GCA/001/605/985/GCA_0016059852_ASM160598v2).

145 The transcripts were identified as lncRNAs or mRNAs using the CPAT, CPC, CNCI and Pfam protein domain

146 databases (Chen et al., 2018). The clean reads were mapped to the Dendrobium officinale reference genome to

147 quantify the gene comparison rate using Bowtie v2.2.3 software (Langmead et al., 2012). The expression of 
148

149

150

151

152

153

154

155

156

157

158

159

160

161

162

163

164

165

166

167

168

transcripts, lncRNAs and mRNAs was calculated using RSEM v1.2.12 software (Li et al., 2011). Differential

expression analysis using the DESeq R package (1.10.1) (Yu et al., 2012). The P-values were adjusted using

Benjamini's approach (Benjamini et al., 1995). Genes with an adjusted P-value $<0.01$ and a $\mid \log _{2}($ fold change) $\mid$

$>1$, as determined with DESeq, were defined as differentially expressed genes.

\section{IncRNA target prediction and annotation}

The cis roles of lncRNAs involve their action on neighbouring target genes. We searched coding genes that

were $10 \mathrm{~kb} / 100 \mathrm{~kb}$ upstream/downstream of lncRNAs and subsequently analysed their function. To understand

the functions of lncRNAs and their targets, Non-Redundant Protein Sequence Database (NR), Nucleotide

Sequence Database (NT), clusters of euKaryotic Orthologous Groups (KOG), Kyoto Encyclopedia of Genes

and Genomes (KEGG), and Swiss-Prot annotations were assigned to the novel and known assembled mRNAs

using Diamond (Buchfink et al., 2015) or Blast (Lobo, 2012). The gene ontology (GO) and InterPro annotations were assigned using Blast2GO (Conesa et al., 2005) and InterProScan5 (Quevillon et al., 2005), respectively. Cytoscape_v3.6.0 software was used for the network mapping of the lncRNAs and their targets.

\section{Secondary metabolite determination}

Freeze-dried grains of D. officinale stems and leaves were extracted as previously described (Li et al., 2018).

The absorbance was measured with a UV-visible spectrophotometer (Evolution 350, Thermo Fisher, MA,

USA), and the wavelengths of polysaccharides, flavonoids and alkaloids were measured at $485 \mathrm{~nm}, 510 \mathrm{~nm}$ and $416 \mathrm{~nm}$, respectively ( Li et al., 2018). The polysaccharide, flavonoid and alkaloid contents in the $D$. officinale stems and leaves were calculated according to established standard curves.

The carotenoids were determined using the method described by Fan et al. (2017). Two grams of fresh $D$.

officinale stems and leaves was weighed and added to $10 \mathrm{~mL}$ of acetone, and the mixture was then incubated in

Peer) reviewing PDF | (2020:06:49729:4:0:NEW 17 Dec 2020) 
169

170

171

172

173

174

175

176

177

178

179

180

181

182

183

184

185

186

187

188

189

the dark until the $D$. officinale material turned white. The absorbance of the sample at $475 \mathrm{~nm}$ was measured using a UV-visible spectrophotometer, and the carotenoid contents were calculated as described by Fan et al. (2017).

\section{Determination of physiological and biochemical indicators}

Determination of superoxide dismutase (SOD) activity. D. officinale leaves (fresh weight) were ground in liquid nitrogen. First, $1 \mathrm{~g}$ of powder was weighed and dissolved in $10 \mathrm{~mL}$ of extract, and the homogenate of $D$. officinale was centrifuged at $8000 \times \mathrm{g}$ and $4{ }^{\circ} \mathrm{C}$ for $15 \mathrm{~min}$. The supernatant was then removed, placed on ice and collected for determination of SOD activity. The activity of SOD in D. officinale leaves was detected using commercial kits (Keming, Suzhou, China) according to the manufacturer's instructions.

Determination of the relative membrane permeability. The relative conductivity of the leaves of $D$. officinale was measured using a conductivity metre (MIK-EC8.0, Meacon, Hanzhou, China). Ten pieces of 1cm-wide leaves were cut and placed in a beaker, and $40 \mathrm{~mL}$ of deionized water was then added. The conductivity was measured immediately after the addition of deionized water $\left(\mathrm{P}_{0}\right)$ and again10 min later $\left(\mathrm{P}_{1}\right)$.

The beaker was then boiled in a boiling water bath for $10 \mathrm{~min}$ and cooled to room temperature; subsequently, deionized water was added to the marked height, and the conductivity $\left(\mathrm{P}_{2}\right)$ was measured. The ratio of the conductivity of the extract before and after boiling indicates the permeability of the cell membrane: relative membrane permeability $(\%)=100\left(\mathrm{P}_{1}-\mathrm{P}_{0}\right) /\left(\mathrm{P}_{2}-\mathrm{P}_{0}\right)$.

Determination of the calmodulin $(\mathrm{CaM})$ content. The CaM samples were prepared according to Wang et al. 2010. Two grams of D. officinale leaves after the different light treatments was weighed and ground with liquid nitrogen to obtain a homogenate. Prechilled cell lysate $\left[50 \mathrm{mmol} \mathrm{L}^{-1}\right.$ Tris- $\mathrm{HCl} \mathrm{pH} 7.4,50 \mathrm{mmol} \mathrm{L}^{-1}$

$\mathrm{NaCl}, 2$ mmol L${ }^{-1}$ EDTA, 1 mmol L ${ }^{-1}$ PMSF, 1 mmol L ${ }^{-1} \beta$-mercaptoethanol, and 1\% (W/W) Triton X-100] 
190

191

192

193

194

195

196

197

198

199

200

201

202

203

204

205

206

207

208

209

210

was added at a ratio of 1:2 (W/V). The cells were disrupted using ultrasonic washing equipment (KQ-

200SPDE, Keqiao, Guangdong, China), boiled at $95^{\circ} \mathrm{C}$ for $5 \mathrm{~min}$, and centrifuged at $10,000 \times \mathrm{g}$ and $4{ }^{\circ} \mathrm{C}$ for 20

min. After discarding the precipitate, the supernatant was the crude extract containing $\mathrm{CaM}$, and the content of

CaM was determined by ELISA. According to the operating instructions provided with the plant calmodulin

ELISA detection kit (Keming, Suzhou, China), the absorbance values of the standard series solutions and

sample solutions were measured with a microplate reader (Multiskan Sky, Thermo Fisher Scientific, MA, USA)

at a wavelength of $450 \mathrm{~nm}$, and the CaM content of $D$. officinale leaves was calculated according to the

established standard curve.

\section{Quantitative real-time PCR (qRT-PCR) analysis}

Total RNA from $D$. officinale leaves after the different light treatments was used for qPCR validation of lncRNAs and mRNAs. Total RNA extraction and determination referred the method described in Li et al.

(2019). The cDNA synthesis, reaction system and procedures, etc. referred the method described by Lin and

Lai. (2010, 2013). The qPCR assays were performed under standard conditions for 40 cycles on a

LightCycler 480 Real-Time PCR System (Roche, Switzerland). Different lncRNA and target genes use

different annealing temperatures. A five-fold dilution gradient of the mixed sample was used to obtain the

standard curve and determine the reliability of the standard curve (Figs. S2-S3). The ACTIN gene was used as

reference gene and the relative lncRNA and mRNA expression levels were calculated with the $2^{-\Delta \Delta \mathrm{Ct}}$ method.

The primers used in this assay are listed in Supplementary Table S2.

\section{Statistical analysis}

The quantitative results concerning the gene expression, physiological and biochemical indexes and functional metabolites of $D$. officinale were obtained from at least three biological replicates. The effects of the 
211 different light treatments on D. officinale were analysed by one-way analysis of variance (ANOVA) followed

212 by Duncan's test using SPSS version 19.0 (IBM, NY, USA). The figures were generated with GraphPad Prism

2136.0 software (GraphPad Software, CA, USA) and Omic-Share online software (Biomarker Technologies, 214 Beijing, China).

\section{RESULTS}

217

218

219

220

221

222

223

224

225

226

227

228

229

230

231

Far-red light can affect the shade-avoidance response of $D$. officinale, and these effects manifest as a series of unique morphological changes. The largest leaf area of $D$. officinale was obtained with the FR4 treatment $\left(125.37 \mathrm{~mm}^{2}\right)$, followed by the FR1 $\left(105.00 \mathrm{~mm}^{2}\right)$ and CK treatments $\left(93.34 \mathrm{~mm}^{2}\right)$. (Table 1; Figs. 1A-1F).

The highest plant height was also obtained with the FR4 $(68.22 \mathrm{~mm})$, followed by the FR1 treatment $(58.20$ $\mathrm{mm})$, whereas the lowest plant height was obtained with the CK treatment $(50.90 \mathrm{~mm})$ (Table 1; Figs. 1A-1F).

The stems of the plants under the FR4 and FR1 treatments were slightly thicker than those obtained with the CK treatment (Table 1; Figs. 1A-1F). Therefore, the results showed that far-red light exerts a significant effect on the height, stem segments and leaf area of $D$. officinale and that the FR4 treatment was the most conducive treatment to increasing the leaf area and accelerating stem elongation in D. officinale.

\section{Sequencing and assembly of transcriptomic data}

This study revealed that an appropriate proportion of far-red light affected the shade-avoidance response of $D$.

officinale by increasing the leaf area and accelerating stem elongation, among other effects, and might thus increase the production of $D$. officinale. To investigate the lncRNAs related to the effects of far-red light on the shade-avoidance response of $D$. officinale, three biological replicate samples after the CK, FR1, and FR4 
232

treatments were subjected to transcriptome sequencing analysis using the Illumina HiSeq platform. After the removal rRNAs and adapter sequences, among other contaminations, an average of $9.66 \mathrm{~Gb}$ of clean data were obtained for each treatment. The RNA-seq data of D. officinale after the different light treatments yielded $65,759,200$ to $88,743,106$ reads (Table 2). The mapping ratio of $D$. officinale reference genomes in all treatments approximately $84.16 \%-86.69 \%$, and uniquely mapping ratio approximately $78.40 \%-80.01 \%$ (Table

2). The sequencing data indicated that the sequencing reads could better match the $D$. officinale reference genome. In addition, the Q20 and Q30 values of the D. officinale samples were higher than 95.47\% (Table 2), demonstrating the high reliability of the $D$. officinale sequencing data.

\section{Analysis of differentially expressed IncRNAs under different light treatments}

The results from the transcriptome sequencing yielded 3,086 new genes, 2,125 differentially expressed genes, 3,770 lncRNAs, and 136 differentially expressed lncRNAs (DE lncRNAs) (Table S3-S11). To study the expression of lncRNAs in D. officinale, all lncRNAs were divided into three categories according to their expression levels: high expression (fragments per kilobase of transcript per million mapped reads (FPKM) > $50)$, moderate expression $(5 \leq \mathrm{FPKM} \leq 50)$ and low expression $(\mathrm{FPKM}<5)$. The results showed that the expression levels of most genes were downregulated, whereas those of a few genes were upregulated (Fig. 2A).

All lncRNAs were analysed, and the DE lncRNAs were identified. The results showed that lncRNAs could be regulated by the different light treatments. The FR1-CK comparison identified a total of 69 DE lncRNAs, including 30 upregulated and 39 downregulated lncRNAs, and in the FR4-CK comparison, 78 DE lncRNAs, including 49 upregulated and 29 downregulated lncRNAs, were identified. Among these lncRNAs, $11 —$ seven upregulated and four downregulated lncRNAs - were found in both the FR1-CK and FR4-CK comparisons (Fig. 2B). A cluster analysis revealed that the 136 DE lncRNAs identified after the different light treatments 
253

254

255

256

257

258

259

260

261

262

263

264

265

266

267

268

269

270

271

272

273

could be divided into six expression patterns (Fig. 2C).

\section{GO analysis of DE IncRNAs in D. officinale}

To better understand the changes in the transcriptome of $D$. officinale under far-red light treatment, a GO analysis was performed using the results from the three different comparisons: FR1-CK, FR4-CK, and FR4-

FR1 (Table 3).

Based on the results from the FR1-CK comparison, a GO analysis of biological processes revealed that the most relevant biological processes of the DE lncRNA target genes included leaf shaping, maturation of 5.8S rRNA, response to brassinosteroids, the ubiquinone- 6 biosynthesis process, and assembly of the large subunit precursor of preribosomes. The most relevant biological processes identified based on the results from the FR4-CK comparison included the ubiquinone-6 biosynthesis process and sucrose biosynthesis process, whereas the analysis of the FR4-FR1 comparison revealed that the most relevant biological process was leaf shaping.

The most enriched cell components obtained from the analysis of the FR1-CK comparison included signal recognition particle endoplasmic reticulum targeting and the HAUS complex, whereas the analyses of the FR4CK and FR4-FR1 comparisons showed that the most relevant cell components were the membrane and cisGolgi networks, respectively.

Based on the results from the FR1-CK comparison, a GO analysis of molecular functions revealed that the most relevant molecular function of the DE lncRNA target genes was translation initiation factor activity. In contrast, the most relevant molecular functions obtained from the analysis of the FR4-CK comparison included O-methyltransferase activity and ureidoglycolate hydrolase activity, and that found from the analysis of the FR4-FR1 comparison was channel activity. 
related to leaf shaping, brassinosteroid synthesis, the ubiquinone- 6 biosynthesis process, membranes, and

methylation, among other processes and components.

277

278

\section{KEGG enrichment analysis of DE IncRNAs in D. officinale}

A KEGG enrichment analysis of the target genes of the DE lncRNAs was also performed (Fig. 3). The most enriched pathways obtained from the analysis of the FR1-CK comparison included brassinosteroid biosynthesis, photosynthesis-antenna proteins, nonhomologous end-joining, tryptophan metabolism and glycerolipid metabolism (Fig. 3A). In contrast, the top five enriched pathways identified from the results of the FR4-CK comparison included stilbenoid, diarylheptanoid and gingerol biosynthesis, phenylalanine metabolism, flavonoid biosynthesis, the AGE-RAGE signalling pathway in diabetic complications, and taurine and hypotaurine metabolism (Fig. 3B). The top five enriched pathways obtained from the FR4-FR1 comparison included brassinosteroid biosynthesis, folate biosynthesis, stilbenoid, diarylheptanoid and gingerol biosynthesis, taurine and hypotaurine metabolism, and sesquiterpenoid and triterpenoid biosynthesis (Fig. 3C).

The top 20 enriched pathways identified from the results of the three comparisons (FR1-CK, FR4-CK, and FR4-FR1) were also analysed, and some of the above-mentioned enriched pathways, such as brassinosteroid biosynthesis and endocytosis, were found in these set of top 20 pathways three comparisons

(Fig. 3D). These results indicated that these pathways exhibit significant differences under the three different treatments (CK, FR1 and FR4).

Moreover, several of the top 20 enriched pathways were also detected in two of the three comparisons (FR1-CK and FR4-FR1), and these included glycerolipid metabolism, glycerophospholipid metabolism, carotenoid biosynthesis and folate biosynthesis (Fig. 3D), which indicates that the FR1 treatment might have a 
295

296

297

298

299

300

301

302

303

304

305

306

307

308

309

310

311

312

313

314

315

significant effect on these pathways. Protein export and glycosylphosphatidylinositol (GPI)-anchor biosynthesis were also identified among the top 20 pathways obtained from the FR1-CK and FR4-CK comparisons (Fig. 3D), which indicated that protein export and GPI-anchor biosynthesis might play important roles in the response to far-red light. The following pathways were among the top 20 enriched pathways identified from the FR4-CK and FR4-FR1 comparisons: stilbenoid, diarylheptanoid and gingerol biosynthesis, flavonoid biosynthesis, phenylpropanoid biosynthesis, plant hormone signal transduction, and taurine and hypotaurine metabolism (Fig. 3D). These results indicate that the FR4 treatment might exert significant effects on these pathways.

Moreover, some of the top 20 enriched pathways were among the top 20 pathways identified from the results of a single comparison; for example, the tryptophan and sulphur metabolism pathways were among the top 20 pathways obtained for the FR1-CK comparison but were not found in the top 20 pathways found for the other two comparisons (Fig. 3D), which indicated that the FR1 treatment might significantly affect tryptophan metabolism and sulphur metabolism. The phenylalanine metabolism, lysine biosynthesis, and circadian rhythm-plant pathways were among the top 20 enriched pathways found only for the FR4-CK comparison (Fig. 3D), which indicated that the FR4 treatment exerts a significant effect on these pathways. Similarly, sesquiterpenoid and triterpenoid biosynthesis were among the top 20 pathways identified from the FR4-FR1 comparison, which indicated that the FR4 treatment has a stronger effect on sesquiterpenoid and triterpenoid biosynthesis than does the FR1 treatment.

\section{Network of far-red light-responsive IncRNAs and their targets}

To further understand the function of DE lncRNAs in the response of D. officinale to far-red light, a lncRNAmRNA interaction network was constructed using Cytoscape software (v.3.6.0) (Figs. 4A-4B; Tables S12-S13). 
Among the lncRNAs obtained from the FR1-CK comparison, MSTRG.60454.1 had the highest number of

317

target genes (18), and these are mainly involved in ribosome biogenesis in eukaryotes, sulphate assimilation, the cytoplasm, membranes, and protein processing in the endoplasmic reticulum (Fig. 4A; Table S12). The expression of MSTRG.41036.9, which is mainly involved in sulphur metabolism, the mitochondrial inner membrane, phosphorylation and RNA transport, exhibited the most significant upregulation (Fig. 4A; Table S12), whereas that expression of MSTRG.70733.3, which is mainly involved in hydrolase activity and activity on ester bonds, showed the most significant downregulation (Fig. 4A; Table S12). In addition, some target genes were also found to be regulated by different lncRNAs identified from the FR1-CK comparison. For example, the genes MA16_Dca022892, MA16_Dca022894, and MA16_Dca022895 are jointly regulated by MSTRG.70733.3 and MSTRG.70733.2 (Fig. 4A). Therefore, the above-mentioned lncRNAs are most likely involved in the effects of FR1 on D. officinale.

Among the lncRNAs obtained from the FR4-CK comparison, MSTRG.26377.2 had the maximal number of target genes (20), and these genes are mainly involved in plant hormone signal transduction, chloroplasts, membranes, and pyruvate metabolism (Fig. 4B; Table S13). MSTRG.19522.1, which is mainly involved in Omethyltransferase activity, methylation, phenylpropanoid biosynthesis, flavonoid biosynthesis, and stilbenoid, diarylheptanoid and gingerol biosynthesis, exhibited the most significantly upregulated expression (Fig. 4B;

Table S13), and the lncRNA that showed the most significant downregulation was MSTRG.36859.3, which is mainly involved in the plant circadian rhythm (Fig. 4B; Table S13). In addition, genes such as MA16_Dca1241, MA16_Dca12410, and MA16_Dca12404 were regulated by MSTRG.33712.1, MSTRG.33712.2, MSTRG.33712.3 and MSTRG.33712.5 (Fig. 4B). Therefore, the above-mentioned lncRNAs are most likely involved in the effects of the FR4 treatment on D. officinale. 
337

338

339

340

341

342

343

344

345

346

347

348

349

350

351

352

353

354

355

356

357

\section{Secondary metabolite contents in $D$. officinale under different light treatments}

The transcriptome sequencing revealed that flavonoid metabolism, alkaloid metabolism, and carotenoid metabolism might affect the shade-avoidance response of $D$. officinale under far-red light. Therefore, the flavonoid, alkaloid and carotenoid contents in the leaves and stems of $D$. officinale were measured after the different far-red light treatments (Fig. 5). The highest flavonoid content in D. officinale leaves was obtained after the FR4 treatment $\left(38.121 \mathrm{mg} \mathrm{g}^{-1}\right)$, followed by the FR1 treatment $\left(32.343 \mathrm{mg} \mathrm{g}^{-1}\right)$, whereas the CK treatment yielded the lowest flavonoid content $\left(29.860 \mathrm{mg} \mathrm{g}^{-1}\right)$ (Fig. 5A; Table S14). The highest flavonoid content in the stems was detected after the FR4 treatment $\left(20.249 \mathrm{mg} \mathrm{g}^{-1}\right)$, followed by the FR1 treatment (18.641 $\left.\mathrm{mg} \mathrm{g}^{-1}\right)$, whereas the lowest content was observed after the CK treatment (13.875 $\left.\mathrm{mg} \mathrm{g}^{-1}\right)$ (Fig. 5B; Table S15). The highest leaf alkaloid content was obtained with the FR4 treatment, followed by the FR1 treatment, whereas the lowest content was found with the CK treatment (Fig. 5C; Table S16). The FR4 and FR1 treatments yielded the highest alkaloid content in the stem segments, whereas the lowest content was detected with the CK treatment (Fig. 5D; Table S17). The highest carotenoid content in the leaves and stems was obtained with the FR4 treatment, followed by the FR1 treatment, whereas the lowest content was found with the CK treatment (Figs. 5E-5F; Tables S18-S19). The highest polysaccharide content of leaves was obtained after the FR4 treatment $\left(108.995 \mathrm{mg} \mathrm{g}^{-1}\right)$, followed by the FR1 treatment $\left(101.683 \mathrm{mg} \mathrm{g}^{-1}\right)$, whereas the CK treatment yielded the lowest polysaccharide content $\left(94.086 \mathrm{mg} \mathrm{g}^{-1}\right)$ (Fig. 5G; Table S20). The highest polysaccharide content of stems was detected after the FR1 treatment $\left(83.294 \mathrm{mg} \mathrm{g}^{-1}\right)$, followed by the FR4 treatment $\left(80.241 \mathrm{mg} \mathrm{g}^{-1}\right)$, whereas the lowest content was observed after the CK treatment $\left(68.811 \mathrm{mg} \mathrm{g}^{-1}\right)$

(Figs. 5H; Tables S21). Therefore, far-red light at appropriate proportions can promote the accumulation of flavonoids, alkaloids, carotenoids and polysaccharides in D. officinale. 
358

359

360

361

362

363

364

365

366

367

368

369

370

371

372

373

374

375

376

377

378

Levels of physiological and biochemical indicators in D. officinale under different light treatments

The transcriptome sequencing revealed that membrane and calcium-transporting ATPase activity might affect the shade-avoidance response of $D$. officinale under far-red light. Therefore, the levels of related physiological and biochemical indicators in the leaves of $D$. officinale under the different far-red light treatments were measured. As an important active oxygen scavenger, SOD can participate in the cell membrane lipid peroxidation defence system. The highest SOD activity was detected with the FR4 treatment, followed by the FR1 and CK treatments (Fig. 6A; Table S22). The highest cell membrane permeability was observed after the FR4 treatment, followed by the FR1 and CK treatments (Fig. 6B; Table S23). The CaM contents obtained with the FR4 and FR1 treatments were higher than that detected after the CK treatment (Fig. 6C; Table S24). These results indicated that the effect of far-red light on $D$. officinale might be closely related to the cell membrane and $\mathrm{Ca}^{2+}$ transduction.

Identification of DE IncRNAs and their targets in D. officinale under different light treatments by qPCR

The qPCR analysis performed in this study revealed 12 groups of lncRNAs and target genes (Fig. 7; Tables S25-S27). Only the expression pattern of MSTRG.58590.1 and its target gene TTA1, MSTRG.19522.1 and its target gene MA16_Dca007905, and MSTRG.38867.1 and its target gene PHYA1 were negatively correlated, which indicated that these three target genes were negatively regulated by their corresponding lncRNAs. MSTRG.63384.1 and its target gene ZSD1, MSTRG.29691.1 and its target gene GGT1_5, MSTRG.66273.1 and its target gene COP1, and MSTRG.48624.1 and its target gene HY5 were positively correlated, which indicating that these four target genes were positively regulated by their corresponding lncRNAs. The remaining lncRNAs and target genes showed different regulatory patterns, which might explain why the target genes were regulated by different members of lncRNA families or other lncRNAs in the plant responses to 
379

380

381

382

383

384

385

386

387

388

389

390

391

392

393

394

395

396

397

398

399

different light conditions.

Some DE lncRNA target genes are related to metabolic pathways. For example, the target gene TAA1 of

MSTRG.58590.1 is a key gene of the tryptophan metabolism pathway, and the expression level of TAA1 after

the FR4 treatment was higher than the levels obtained after the FR1 and CK treatment (Fig. 7A). The target

gene CCD4 of MSTRG.22972.1 and the target gene ZSD1 of MSTRG.63384.1 are key genes of the carotenoid

synthesis pathway. The expression levels of CCD4 and ZSD1 under far-red light were higher than those

obtained after the CK treatment (Figs. 7B-7C). Some DE lncRNA target genes are related to hormone signal

transduction. The target gene CYP90A1 of MSTRG.25820.7 is involved in the brassinolide signal transduction

pathway in D. officinale. The highest expression of CYP90A1 was obtained with the FR4 treatment, followed

by the FR1 and CK treatments, which indicated that brassinolide signal transduction plays an important role in

the shade-avoidance response of $D$. officinale under far-red light (Fig. 7E). Some DE lncRNA target genes are

closely related to DNA methylation. For example, the MSTRG.19522.1 target gene MA16_Dca007905

functions in O-methyltransferase activity (Fig. 7H). In addition, some DE lncRNA target genes are also related

to far-red light signal transduction; for example, MSTRG.38867.1 and its target gene PHYA1,

MSTRG.69319.1 and its target gene SPA1, MSTRG.66273.1 and its target gene COP1, MSTRG.48624.1 and

its target gene HY5 MSTRG.71071.1 and its target gene PIF3 might be involved in the signal transduction pathways of $D$. officinale induced in response to far-red light (Figs. 7I-7L).

\section{DISCUSSION}

\section{Cell signalling perception and conduction in D. officinale under far-red light}

The GO analysis revealed that the lncRNAs identified from the FR4-CK comparison were significantly 
400

401

402

403

404

405

406

407

408

409

410

411

412

413

414

415

416

417

418

419

420

enriched in the membrane, and the lncRNAs identified from the FR1-CK and FR4-CK comparisons were significantly enriched in calcium-transporting ATPase activity (Table 3). The physiological and biochemical experiments also revealed that the activity of SOD, the cell membrane permeability and the CaM content obtained after far-red light treatment were higher than those obtained after the control treatment (Figs. 6A-6C).

Therefore, the shade-avoidance response of $D$. officinale to far-red light might be closely related to the cell membrane and $\mathrm{Ca}^{2+}$ transduction. The exposure of plant cells to external factors induce corresponding changes in the cell wall components. Elements that perceive environmental changes are distributed in the periplasmic interval between the cell wall and the cell membrane, and these elements can induce increases in the $\mathrm{Ca}^{2+}$ concentration in the cytosol. The cell membrane carries information concerning external factors as well as cell information and participates in energy and material exchange. Moreover, the cell membrane can maintain the stability of the intracellular environment and provide order to physiological and metabolic pathways in plants

(Graier et al., 2010). As an important secondary messenger in plants, $\mathrm{Ca}^{2+}$ can respond to external factors (Moeder et al., 2019). Under the influence of external factors, the $\mathrm{Ca}^{2+}$ concentration of plants increases instantaneously and can be sensed by various $\mathrm{Ca}^{2+}$ sensors and binding proteins, including calmodulin (CaM), calcium-dependent protein kinases (CDPKs) and calcineurin B-like proteins (CBLs) (González et al., 2018).

$\mathrm{CaM}$ can act on various organelles and their constituents, such as the cell membrane, vacuole, and nucleus, and is involved in the regulation of important physiological and biochemical processes and secondary metabolism in plants (Yamashita et al., 2018). For example, CaM plays an important role in the reproductive growth of plants, and its gene family members CaM2 and CaM7 can promote the growth of pollen tubes. Wang et al. (2010) reported that $\mathrm{CaM}$ is involved in potato glycoside alkaloid metabolism in response to light.

IncRNAs involved in some metabolic pathways influence the effects of far-red light on the shade- 
421

422

avoidance response of $D$. officinale

The KEGG analysis revealed that the following pathways were among the top 20 pathways enriched in the lncRNAs identified from the FR1-CK and FR4-CK comparisons: phenylalanine metabolism, phenylpropanoid biosynthesis, tryptophan metabolism, stilbenoid, diarylheptanoid and gingerol biosynthesis, flavonoid biosynthesis, and carotenoid biosynthesis (Fig. 3). The phenylalanine metabolism and phenylpropanoid biosynthesis pathways are upstream of tryptophan metabolism, stilbenoid, diarylheptanoid and gingerol biosynthesis, flavonoid biosynthesis, and carotenoid biosynthesis ( $\mathrm{Li}$ et al., 2019). Physiological and biochemical experiments also verified that the contents of flavonoids, carotenoids, alkaloids, and polysaccharides after the far-red light treatments were higher than those obtained after the control treatment.
The results of this study showed that far-red light can promote the accumulation of flavonoids in $D$.

officinale because phytochrome A $(P H Y A)$ is an essential component in far-red light-induced flavonoid synthesis ( Li et al., 2014). Plant flavonoid synthesis is related to the response to external factors, including farred light (Li et al., 2014), and flavonoids protect plants from oxidative damage by absorbing far-red light and removing reactive oxygen species (Xie et al., 2015). Far-red light induces plant photomorphogenesis and flavonoid accumulation through the inhibition of CUL4-DDB1 ${ }^{\mathrm{COP} 1 / \mathrm{SPA}}$ by $P H Y A$ to stabilize the degradation of its substrate (Maier et al., 2015).

The results of this study also showed that tryptophan metabolism (upstream of alkaloid metabolism) was significantly enriched (Fig. 3), and the expression of TAA1 (DE lncRNA target gene), which is involved in the synthesis of metabolites, was upregulated in D. officinale under far-red light treatment (Fig. 7). Studies on Arabidopsis have revealed that TAA1 is involved in catalysing the reaction of tryptophan and pyruvate to produce etodolac and, subsequently, auxin (Won et al., 2011). The discovery of TAA1 confirmed the existence 
442 of a pathway for the synthesis of indoleacetic acid (IAA) in plants. In addition, after perceiving shaded signals,

443 Arabidopsis plants might rapidly increase their level of IAA through the regulation of $T A A 1$ and thereby

444 rapidly induce the expression of IAA response genes and downstream genes, which leads to rapid hypocotyl

445 extension, among other effects that form part of the shade-avoidance phenomena (Won et al., 2011). Therefore,

446 tryptophan metabolism might affect the shade-avoidance response of $D$. officinale under far-red light.

447 The results of this study also showed that carotenoid metabolism was significantly enriched in $D$.

448 officinale (Fig. 3). The expression of both the CCD4 and ZSD1 genes (DE lncRNA targets) was upregulated

449 under far-red light treatment (Fig. 7). CCD4 is a key gene in apocarotenoid synthesis, and ZSD1 is a key gene

in ABA synthesis. Related studies have shown that strawberry leaves have a high cucurbitacin content under

low R:FR ratio (Sun et al., 2010). Carotenoids are an indispensable class of pigments in higher plants. These

compounds act as antenna pigments for photosynthesis and transmit captured light energy to chlorophyll.

Carotenoids also function in light protection and the scavenging of free radicals and thus protect plant tissues

from damage induced by strong light (Sun et al., 2010). Carotenoids are also synthetic precursors of

phytohormones such as ABA, strigolactone and lactone, which play important roles in plant growth and

that can protect cells from oxidative damage and maintain the normal function of the cellular system ( Liu et al.,

2016). Therefore, carotenoid metabolism might affect the shade-avoidance response of $D$. officinale under farred light.

The KEGG analysis revealed the AGE-RAGE signaling pathway in diabetic complications as among the top 5 pathways enriched in the lncRNAs identified from the FR4-CK comparisons (Fig. 3D). The AGE-RAGE signaling pathway plays an important role in the occurrence and development of diabetic nephropathy (Yang et 
463

464

465

466

467

468

469

470

471

472

473

474

475

476

477

478

479

480

481

482

483

al., 2019). Modern pharmacological studies have shown that Coptis polysaccharide can significantly reduce the expression of HU-VEC on mRNA and protein RAGE induced by AGEs (Yin et al., 2012). It is confirmed that Coptis polysaccharide might treat diabetic nephropathy by blocking the AGE-RAGE signaling pathway (Yin et al., 2012). Yuan et al. (2017) confirmed that puerarin has a significant inhibitory effect on the formation of AGEs in vivo and in vitro, and reduce the expression of RAGE on mRNA in the kidney tissue of streptozotocin-induced diabetic rats to improve diabetic nephropathy (Shen et al., 2009). Related studies also show that berberine can significantly affect the AGE-RAGE signaling pathway, and its important target factor is AGEs (Qiu et al., 2017). The AGE- RAGE signaling pathway is significantly enriched in the shadeavoidance response of D.officinale under far-red light, which may be closely related to the metabolic pathways of polysaccharides, flavonoids and alkaloids.

In addition, the KEGG analysis revealed taurine and hypotaurine metabolism as among the top 20 pathways enriched in the lncRNAs identified from the FR4-CK and FR4-FR1 comparisons (Fig. 3D). Hypotaurine is an $\mathrm{S}^{2-}$ scavenger, which plays an important role in sulfur metabolism in plants (Shan et al., 2011). $\mathrm{H}_{2} \mathrm{~S}$ is produced by the decomposition of cysteine in plants, and cysteine is an important hub of the sulfur conversion pathway. Cysteine can also be broken down to produce pyruvic acid, which is an important substance in carbon metabolism (Shan et al., 2011). Studies on corn have found that $\mathrm{H}_{2} \mathrm{~S}$ can promote the synthesis of triterpenoids by regulating sulfur and carbon metabolism(Capaldi et al., 2015). During the response of $D$. officinale to far-red light, the expression of $\gamma$-glutamyl transpeptidase (GGT1, DE lncRNA target gene), which is involved in the taurine and sulfinate metabolic pathways, was significantly upregulated. Increase in the content of 5-glutamyl taurine increased were accompanied by decreases in the taurine and sulfinate (a $\mathrm{S}^{2-}$ scavenger) contents and increase in the content of $\mathrm{S}^{2-}$. Increased $\mathrm{S}^{2-}$ concentrations might affect 
484

the mevalonate pathway as well as the synthesis of alkaloids, carotenoids, and fatty acids, among other metabolites (Mugford et al., 2011). Therefore, taurine and hypotaurine metabolism might play an important role in the shade-avoidance response of $D$. officinale under far-red light.

IncRNAs involved in hormone signal transduction influence the shade-avoidance response of $D$.

\section{officinale to far-red light}

During the response of $D$. officinale to far-red light, the functions of target genes of DE IncRNAs involve plant hormone signal transduction (Fig. 3; Table 3). Light signals are closely related to hormones during the process of regulating plant growth and development (Warpeha et al., 2015). The plant hormone levels exhibit changes depending on the light conditions and photosensitive pigment activity, which indicates that light signals can participate in plant hormone signal transduction and thereby regulate plant growth and development and the activity of metabolic pathways ( $\mathrm{Li}$ et al., 2018). The plant shade-avoidance response also involves hormone regulation, and the GO and KEGG enrichment analyses performed in this study showed that BR, auxin and cytokinin were significantly enriched in D. officinale under far-red light (Fig. 3; Table 3).

BR can promote plant cell elongation, division and vascular bundle development. BR plays an important role in regulating the light response of plants (Nafie et al., 2015). BR can also activate the antioxidant enzyme protection system and thereby eliminates excessive harmful free radicals induced as a result of external factors and improves the ability of plants to respond to external factors (Oh et al., 2012). The results of this study showed that the expression of BR signalling kinase ( $B S K$, DE lncRNA target gene), a gene downstream of BRI1, was significantly upregulated by the FR4 treatment (Fig. 7). Related studies have shown that brassinosteroid insensitive 1 (BRI1) is a BR receptor with kinase activity. The cultivation of Arabidopsis plants under far-red light rapidly upregulates the expression of BRI1. Arabidopsis plants without BRI1 function 
505

506

507

508

509

510

511

512

513

514

515

516

517

518

519

520

521

522

523

524

525

comprise significantly fewer cells and are severely dwarfed. These findings show that $B R I 1$ plays an important role in the light-induced elongation of Arabidopsis (Ouyang et al., 2011).

Auxin (IAA) is a hormone that regulates the direction of plant growth resulting from specific regions of cells presenting increased activity and division. The main role of IAA is to regulate plant growth, and this hormone specifically stimulates the vertical growth of stem cells and inhibits the lateral growth of root cells (Gan et al., 2013). Studies in Arabidopsis have shown that the TAA1, indole-3-acetic acid inducible (IAA), and pin-formed $(P I N)$ genes participate in the shade-avoidance response (Yi et al., 2008). Moreover, the TAA1 mutant sav3 exhibits the hypoxia hypothesis because the hypocotyl elongated faster than that of the wild type under a low R:FR ratio. $I A A$ is an early auxin response gene and encodes a transcription factor that regulates auxin expression. Researchers have found that the expression of IAA19 under low R:FR conditions is threefold higher than that under white light (Pierik et al., 2009). The results of this study showed that the expression levels of TAA1 and IAA (DE lncRNA targets) in the auxin signalling pathway were significantly upregulated under far-red light (Fig. 7); thus, these genes might be key players in the effects of far-red light on the shadeavoidance response of $D$. officinale.

Cytokinins can promote cell division and differentiation, eliminate apical dominance, and promote lateral bud growth. Related reports indicate that cytokinins are involved in the shade-avoidance response of $A$. thaliana, and the genes involved in the shade-avoidance response include cytokinin oxidase $5(C K X 5)$ and cytokinin oxidase 6 (CKX6), both of whose encoded proteins can degrade key enzymes involved in cytokinin synthesis and regulate the cytokinin levels. Researchers using the $\beta$-glucuronidase (GUS) staining method found that the expression of AtCKX6::GUS is significantly increased in Arabidopsis protolayer cells of the leaf primordium under low R:FR conditions (Carabelli et al., 2007). The results of this study showed that the 
526

527

528

529

530

531

532

533

534

535

536

537

538

539

540

541

542

543

544

545

546

expression of $A R R-A$ (which encodes a two-component response regulator of the ARR-A family, DE lncRNA

target gene) in the cytokinin signalling pathway was significantly upregulated under far-red light (Fig. 7),

which might affect the shade-avoidance response of $D$. officinale under far-red light.

In summary, lncRNAs might be involved in the signal transduction of BR, auxin and cytokinins and thereby influence the effects of far-red light on the shade-avoidance response of $D$. officinale.

\section{DNA methylation affects the shade-avoidance response of $D$. officinale under far-red light}

The functions of some target genes of DE lncRNAs involve O-methyltransferase activity, and the GO analysis revealed that the lncRNAs identified in the FR4-CK comparison were significantly enriched in this activity

(Table 3). DNA methylation is an important epigenetic modification that plays an important role in shaping the chromatin structure and regulating gene expression. DNA methylation is involved in many biological activities, such as embryonic development, abiotic stress, light regulation, and tissue-specific gene expression (Vanyushin et al., 2011; Kuo et al., 2015). Kuo et al. (2015) used whole-genome expression and DNA methylation profiles to study the effects of red and far-red light on genes regulated by the secondary metabolic pathways in agarwood and found that the expression of small RNA-regulated genes might occur through RNA-directed DNA methylation. Laser radiation at a certain frequency not only causes changes in the DNA methylation patterns in rice (O. sativa L.) but also alters the expression of epigenetically modified genes that function in the maintenance, structure and state of chromatin, such as causing changes in the expression of methyltransferases, glycosidases and important enzymes involved in the short interfering RNA (siRNA) production pathway. Moreover, a correlation analysis confirmed that these changes are related to methylation (Li et al., 2017). Wang et al. (2010) treated geminated seeds of orthogonal sorghum F1 hybrids and pure parents with a low-intensity laser and found that the sorghum F1 hybrids and corresponding parents presented 
547 some changes in the level and pattern of DNA methylation. Therefore, O-methyltransferase activity might play

548 an important role in the shade-avoidance response of $D$. officinale under far-red light.

549 IncRNAs involved in the signal transduction pathways responsible the effects of far-red light on the

550

551

552

553

554

555

556

557

558

559

560

561

562

563

564

565

566

567

shade-avoidance response of $D$. officinale

Based on previous studies on the signal transduction pathways related to far-red light (Kang et al., 2009;

Zheng et al., 2013; Sharkhuu et al., 2014), this study established a regulatory network for the shade-avoidance response of D. officinale to far-red light (Fig. 8).

In the signal transduction pathway for the effects of far-red light in the shade-avoidance response of $D$. officinale, DE lncRNA MSTRG.38867.1 might act through the PHYA target gene, whereas the DE lncRNAs MSTRG.64192.1, MSTRG.64193.2 and MSTRG.64207.1 might act through the target gene PHYB (Fig. 8). Photosensitive pigments, which include $\mathrm{Pr}$ and $\mathrm{Pfr}$ phytochromes, play an important role in the shadeavoidance response. After absorbing one photon, Pr is converted to Pfr, and after absorbing another photon, it is converted back to $\operatorname{Pr}$ (Chen et al., 2018). PHYB is a photoreceptor involved in most shade-avoidance reactions. Pfr is transported from the cytoplasm to the nucleus under shaded conditions such that it can more easily bind with transcription factors to exert its biological activity. $P H Y B$ can also sense a low R:FR ratio in shaded environments and can promote the conversion of Pfr to Pr (Julia et al., 2010). Studies of A. thaliana have shown that $P H Y B$ plays an important role in dark reversal and is a good sensor of light irradiance during early seed development (Julia et al., 2010). Because the light conversion response is slow under shaded conditions, the dark reversal of Pfr can stably promote the conversion of Pfr to Pr. Although $P H Y A$ is not sensitive to the R:FR ratio, it is a sensitive sensor of changes in light irradiance during the shade-avoidance response, potentially because most photomorphological responses require the presence of Pfr in the nucleus 
568

569

570

571

572

573

574

575

576

577

578

579

580

581

582

583

584

585

586

587

588

(Rausenberger et al., 2011). In addition, PHYA is very important for the conversion of the dark phytochrome form to the light phytochrome form under shaded conditions, which results in a process called de-etiolation. ${ }^{62}$

The DE lncRNA MSTRG.71071.1 might participate in the signal transduction pathway underlying the shade-avoidance response of $D$. officinale to far-red light through the target gene PIF3 (Fig. 8). The main characteristic of PIFs is that they can interact with the Pfr form of the photosensitive pigment and thereby regulate the growth of stems as part of the shade-avoidance response (Zhou et al., 2012). Seven members of this subfamily, PIF1/PIF3-LIKE 5 (PIL5), PIF3, PIF4, PIF5/PIL6, PIF6/PIL2, PIF7, and PIF8, interact directly with the conserved N-terminal sequence of $P H Y B$, whereas two members, PIF1/PIL5 and PIF3, interact with phyA (Zhou et al., 2012; Liu et al., 2011). The shade-avoidance response under low R:FR conditions requires the participation of PIF3, PIF4, PIF5, and PIF7 (Zhou et al., 2012; Liu et al., 2011). For example, PIF3 functions mainly to control the morphological phenotype of plants and participates in some biochemical pathways involved in the light response process. Under high R:FR conditions, Pfr induces the phosphorylation of PIF7 and reduces the binding of PIF7 to the cotarget promoter to induce negative regulation. When plants are transferred to low R:FR conditions, PIF7 increases its binding to its target promoter and thereby exerts its effect (Zhou et al., 2012; Liu et al., 2011). These results indicate that the transition from a high to a low R:FR ratio under shaded conditions will reduce the active form of PHYB and thereby increase the activity of PIFs. An increased activity of PIFs will in turn promote the growth of plants during the shade-avoidance response (Lau et al., 2010).

The DE lncRNAs MSTRG.32929.1, MSTRG.58888.1, and MSTRG.66273.1 might act through the target gene COP1, and the DE lncRNA MSTRG.69319.1 might act through the target gene SPA1 and participate in the signal transduction pathway underlying the shade-avoidance response of $D$. officinale to far-red light (Fig. 
589

590

591

592

593

594

595

596

597

598

599

600

601

602

603

604

605

606

607

608

609

8). COP1, a conserved RING finger E3 ubiquitin ligase, is involved in many biological processes, including plant growth and development and metabolic processes (Pacin et al., 2013). During emergence, plant seedlings undergo a transition from darkness to light. At this time, the expression of $c r y 1, P H Y A$, and $P H Y B$ is induced by light, and COP1 slowly moves from the nucleus to the cytoplasm. SPA1 acts as a cofactor to regulate COP1 ubiquitin ligase activity (Pacin et al., 2013). In Arabidopsis, SPA proteins can self-associate with each other and form COP1/SPA heteropolymers, as reflected via various comparisons with COP1 (Zhu et al., 2008).

These COP1/SPA complexes form part of the CULLIN 4-DAMAGED DNA-BINDING 1 ubiquitin E3 ligase complex (CUL4-DDB1 ${ }^{\mathrm{COP} 1 / \mathrm{SPA})}$ ) and mediate substrate recognition (Lau et al., 2010). Several positive regulators of the light response, including $H Y 5, H Y H$, long after far-red light 1 (LAF1), and $H F R 1$, are

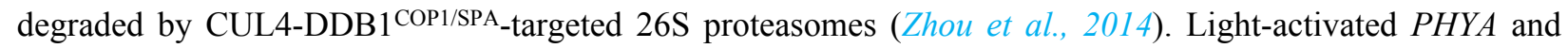
PHYB downregulate the E3 ubiquitin ligase activity of CUL4-DDB1 ${ }^{\mathrm{COP} 1 / \mathrm{SPA}}$ and thereby stimulate the light response by stabilizing these positive regulators.

The DE lncRNA MSTRG.48624.1 might act through the target gene HY5, whereas the DE lncRNAs MSTRG.65167.1, MSTRG.65169.4, and MSTRG.65169.5 may act through the target gene LAF1 and participate in the signal transduction pathway underlying the shade-avoidance response of $D$. officinale to farred light (Fig. 8). HY5 has an extremely wide spectrum of effects, including those caused by far-red, red, blue, and UV-B light (Lockhart et al., 2014). The results of this study showed that the HY5 protein level was positively correlated with the degree of photomorphogenesis (Rodrigo et al., 2016). Moreover, it has been found that the target genes of HY5 are related mainly to physiological processes such as photomorphogenesis, anthocyanin and chlorophyll synthesis, and lateral root development (Sara et al., 2017). HFR1 cannot directly interact with $P H Y A$ or $P H Y B$, but HFR1/PIF3 dimers preferentially interact with $P H Y A$ and $P H Y B$ via Pfr 
610 (Jang et al., 2013). HFR1 regulates the expression of target genes by interacting with other basic helix-loop-

611 helix (bHLH)-type transcription factor PIFs (Hornitschek et al., 2009). For example, HFR1 prevents a strong

612 shade-avoidance response by forming a heterodimer that does not bind to DNA with PIF4 or PIF5. PIF4 and

613 PIF5 are two bHLH-type transcription factors that can directly regulate the expression of marker genes of the

614 shade-avoidance response (Hornitschek et al., 2009). HFR1 and PIF1 interact to inhibit the binding of PIF1

615 and downstream target gene promoters and regulate seed development (Shin et al., 2013). LAF1 regulates the

616 PHYA signalling pathway and also directly interacts with $H F R 1$, and this interaction can inhibit the

ubiquitination of $C O P 1$, stabilize $H F R 1$ and $L A F 1$, and promote the transduction of $P H Y A$ signals, which

ultimately affects plant photomorphogenesis (Jang et al., 2013).

\section{CONCLUSION}

This study provides the first demonstration of the effects of far-red light on lncRNAs involved in the shade-

avoidance response of $D$. officinale through an RNA-seq analysis. We found that an appropriate proportion of

far-red light can increase the leaf area, accelerate stem elongation, and thereby increases the production of $D$.

officinale. Based on the transcriptomic, physiological and biochemical analyses, we revealed that lncRNAs

involved in some metabolic pathways (i.e., flavonoid metabolism, alkaloid metabolism, carotenoid metabolism

and polysaccharide metabolism) influence the effects of far-red light on the shade-avoidance response of $D$.

officinale. The effect of far-red light on $D$. officinale might be closely related to cell signalling perception and 
631 Cytoscape analysis and previous studies on far-red light-related signal transduction pathways, this study also

632

633

634

635

636

637

638

639

640

641

642

643

644

645

646

647

648

649

650

651

found that some lncRNAs might participate in the far-red light signalling network through their target genes

and thus regulate the shade-avoidance response of $D$. officinale. These findings provide new insights into the

shade-avoidance response of $D$. officinale under far-red light and will be helpful for generating new ideas for the high-yield production of medicinal components of D. officinale.

\section{ACKNOWLEDGEMENTS}

We thank American Journal Experts for editing the English text of a draft of this manuscript.

\section{ADDITIONAL INFORMATION AND DECLARATIONS}

\section{Funding}

This work was funded by National Natural Science Foundation of China (31501802), Natural Science

Foundation of Fujian Province (2020J01377), Education research project for young and middle-aged teachers

in Fujian (JAT190696), Sanming University Scientific Research Foundation for High-level Talent (18YG01, 18YG02, 19YG06), 2019 and 2020 Special Commissioner of Science and Technology of Fujian Province.

\section{Competing Interests}

The authors declare that they have no competing interests.

\section{Author Contributions}

- Hansheng Li performed the experiments, analyzed the data, prepared figures and/or tables, authored or reviewed drafts of the paper, and approved the final draft.

- Wei Ye performed the experiments, analyzed the data, authored or reviewed drafts of the paper, and 
652

653

654

655

656

657

658

659

660

661

662

663

664

665

666

667

668

669

670

671

672

approved the final draft.

- Yaqian Wang analyzed the data, authored or reviewed drafts of the paper, and approved the final draft.

- Xiaohui Chen analyzed the data, authored or reviewed drafts of the paper, and approved the final draft.

- Yan Fang analyzed the data, authored or reviewed drafts of the paper, and approved the final draft.

- Gang Sun conceived and designed the experiments, analyzed the data, prepared figures and/or tables, authored or reviewed drafts of the paper, and approved the final draft.

\section{Supplemental Information}

Figure S1 The electrophoretogram of total RNA of $D$. officinale samples under different light treatments

Figure S2 The standard curves of lncRNA and targets

Figure S3 The Melt curve plots of lncRNA and targets

Table S1 The concentration and A260/A280 ratios of total RNA of samples

Table S2 Primers information used for real-time PCR analysis of D. officinale genes

Table S3 Sequencing result data statistics under different light treatments

Table S4 The related information of new genes

Table S5 The related information of differentially expressed genes in the FR1-CK

Table S6 The related information of differentially expressed genes in the FR4-CK

Table S7 The related information of differentially expressed genes in the FR4-FR1

Table S8 The related information of lncRNAs

Table S9 The related information of differentially expressed lncRNAs in the FR1-CK

Table S10 The related information of differentially expressed lncRNAs in the FR4-CK

Table S11 The related information of differentially expressed lncRNAs in the FR4-FR1

Peer] reviewing PDF | (2020:06:49729:4:0:NEW 17 Dec 2020) 
673 Table S12 Annotation of differentially expressed lncRNAs in the FR1-CK

674 Table S13 Annotation of differentially expressed lncRNAs in the FR4-CK

675 Table S14 Flavonoid contents of leaves in D. officinale under different light treatments

676 Table S15 Flavonoid contents of stems in D. officinale under different light treatments

677 Table S16 Alkaloid contents of leaves in D. officinale under different light treatments

678 Table S17 Alkaloid contents of stems in D. officinale under different light treatments

679 Table S18 Carotenoid contents of leaves in D. officinale under different light treatments

680 Table S19 Carotenoid contents of stems in D. officinale under different light treatments

681 Table S20 Polysaccharide contents of leaves in D. officinale under different light treatments

682 Table S21 Polysaccharide contents of stems in D. officinale under different light treatments

683 Table S22 SOD content of leaves in D. officinale under different light treatments

684 Table S23 Relative membrane permeability of leaves in D. officinale under different light treatments

685 Table S24 CaM content of leaves in D. officinale under different light treatments

686 Table S25 The Ct mean of DE lncRNAs and their targets in D. officinale under different light treatments

687 Table S26 The efficiency value of DE lncRNAs and their targets in D. officinale under different light

688 treatments

689 Table S27 The relative expression of DE lncRNAs and their targets in D. officinale under different light

690 treatments

691

692 REFERENCES

693 Amanda SL, Herrera LA, Maloof JN. 2016. Tomato phyE Is Required for Shade Avoidance in the Absence 
694

695

696

697

698

699

700

701

702

703

704

705

706

707

708

709

710

711

712

713

714

of phyB1 and phyB2. Frontiers in Plant Science 7: 1-9.

Benjamini Y, Hochberg Y. 1995. Controlling the false discovery rate: a practical and powerful approach to multiple testing. $J$ R Statist Soc B 57:289-300.

Buchfink B, Xie C, Huson DH. 2015. Fast and sensitive protein alignment using DIAMOND. Nat Methods 12(1):59-60.

Capaldi FR, Gratão PL, Reis AR, Lima LW, Azevedo RA. 2015. Sulfur metabolism and stress defense responses in plants. Tropical Plant Biology 8(3): 60-73.

Carabelli M, Possenti M, Sessa G, Ciolfi A, Sassi M, Morelli G, Ruberti I. 2007. Canopy shade causes a rapid and transient arrest in leaf development through auxin-induced cytokinin oxidase activity. Genes \& Development 21(15): 1863-1868.

Chen S , Andrea M M , Christina L, Jakub K, Wolfgang G, Jörg M, Jon H. 2018. 3D Structures of Plant Phytochrome A as Pr and Pfr From Solid-State NMR: Implications for Molecular Function. Frontiers in Plant Science 9:498-512.

Chen W, Semiconductors OO. 2015. Application of $730 \mathrm{~nm}$ far red light LEDs in horticulture lighting. China Light \& Lighting 8: 29-31.

Chen Y, Li X, Su L, Chen X, Zhang S, Xu X, Zhang Z, Chen Y, Xu X, Lin Y, Lai Z. 2018. Genome-wide identification and characterization of long non-coding RNAs involved in the early somatic embryogenesis in Dimocarpus longan Lour[J]. BMC Genomics 19(1): 905-824.

Conesa A, Götz S, García-Gómez JM, Terol J, Talón M, Robles M. 2005. Blast2GO: a universal tool for annotation, visualization and analysis in functional genomics research. Bioinformatics 21(18):3674-6.

Dai ZH, Fan LY, Zhu H, Teng JB, Li P, Li PF, Huang PL. 2018. Effects of Phytohormones and 
Sunshading on Polysaccharide Content in Dendrobii officinalis. Molecular Plant Breeding 23: 255-262.

Fan ZQ, Zhi-Ying LI, Dong MH, Zhou B, Ouyang YX, Yang LY, Li SL. 2017. Carotenoid-producing yeasts resources in Fuxian Lake, Yunnan. Microbiology China 44(2): 296-304.

Gan D, Zhuang D, Ding F, Yu Z, Zhao Y. 2013. Identification and expression analysis of primary auxinresponsive Aux/IAA gene family in cucumber (Cucumis sativus). Journal of Genetics 92(3): 513-521.

Garima B, Neetu G, Shailesh S, Santosh U, Kashmir S. 2017. Present scenario of long non-coding RNAs in plants. Non Coding Rna 3(2): 16-37.

González A, Sáez CA, Moenne A. 2018. Copper-induced activation of TRPs and VDCCs triggers a calcium signature response regulating gene expression in Ectocarpus siliculosus. peer journal 6(6): e4556.

Graier WF, Paltauf-Doburzynska J, Hill BJF, Fleischhacker E, Hoebel BG, Kostner GM, Sturek M. 2010. Submaximal stimulation of porcine endothelial cells causes focal $\mathrm{Ca}^{2+}$ elevation beneath the cell membrane. The Journal of Physiology 506(1): 109-125.

Hornitschek P, Lorrain S, Zoete V, Michielin O, Fankhauser C. 2009. Inhibition of the shade avoidance response by formation of non-DNA binding bHLH heterodimers. Embo Journal 28(24): 3893-3902.

Hou JN, Wang Z, Shang WQ, Song-Lin HE, Yang B. 2013. Effects of different light quality ratios of CCFL on multiplication of Dendrobium officinale PLB and growth of plantlets in vitro. Journal of Henan Agricultural Sciences 42(1): 86-89.

Jang IC, Henrigues R, Chua NH. 2013. Three transcription factors, HFR1, LAF1 and HY5, regulate largely independent signaling pathways downstream of phytochrome A. Plant Cell physiol 54(6): 907-16.

Julia R, Andrea H, Stefan K, Daniel K, Jens T, Ferenc N. 2010. An integrative model for phytochrome B mediated photomorphogenesis: from protein dynamics to physiology. Plos One 5(5): e10721. 
736

737

738

739

740

741

742

743

744

745

746

747

748

749

750

751

752

753

754

755

756

Kang CY, Lian HL, Wang FF, Huang JR, Yang HQ. 2009. Cryptochromes, phytochromes, and COP1 regulate light-controlled stomatal development in Arabidopsis. Plant Cell 21(9): 2624-2641.

Kasulin L, Agrofoglio Y, Botto JF. 2013. The receptor-like kinase ERECTA contributes to the shadeavoidance syndrome in a background-dependent manner. Annals of Botany, 111(5): 811-819.

Kuo TC, Chen CH, Chen SH, Lu IH, Chu MJ, Huang LC, Lin CY, Chen CY, Lo HF, Jeng ST, Chen LF. 2015. The effect of red light and far-red light conditions on secondary metabolism in Agarwood. BMC Plant Biology 15(1): 139-148.

Kutschera U, Briggs WR. 2013. Seedling development in buckwheat and the discovery of the photomorphogenic shade-avoidance response. Plant Biology 15(6): 931-940.

Langmead B. 2012. Fast gapped-read alignment with Bowtie 2. Nature Methods 9(4): 357-359.

Lau OS, Xing WD. 2010. Plant hormone signaling lightens up: integrators of light and hormones. Current Opinion in Plant Biology 13(5): 571-577.

Li B. 2011. RSEM: accurate transcript quantification from RNA-Seq data with or without a reference genome. BMC Bioinformatics 12(1): 323-323.

Li H, Chen X, Wang Y, Yao D, Lin Y, Lai Z. 2018. Exploration of the effect of blue light on microRNAs involved in the accumulation of functional metabolites of longan embryonic calli through RNAsequencing. Journal of the Science of Food \& Agriculture 99(4): 1533-1547.

Li H, Lyu Y, Chen X, Wang C, Yao D, Ni S, Lin Y, Chen Y, Zhang Z, Lai Z. 2019. Exploration of the effect of blue light on functional metabolite accumulation in longan embryonic calli via RNA sequencing. International Journal of Molecular Sciences 20(2):441-464.

Li S, Xia Q, Fang W, Yu X, Jian M, Kou H, Lin X, Gao X, Liu B. 2017. Laser irradiation-induced DNA 
757

758

759

760

761

762

763

764

765

766

767

768

769

770

771

772

773

774

775

776

777

methylation changes are heritable and accompanied with transpositional activation of mPing in Rice.

Frontiers in Plant Science 8: 363.

Li T, Jia KP, Lian HL, Yang X, Li L, Yang HQ. 2014. Jasmonic acid enhancement of anthocyanin accumulation is dependent on phytochrome A signaling pathway under far-red light in Arabidopsis. Biochemical \& Biophysical Research Communications 454(1):78-83.

Lin Y, Lai Z, 2010. Reference gene selection for qPCR analysis during somatic embryogenesis in longan tree. Plant Sci 178 (4): 359-365.

Lin Y, Lai Z, 2013. Superoxide dismutase multigene family in longan somatic embryos: a comparison of

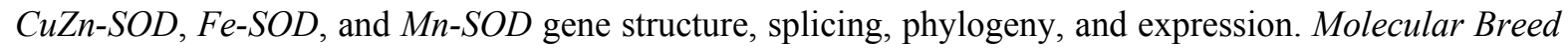
32 (3): 595-615.

Liu J, Jung C, Xu J, Wang H, Deng S, Bernad L, Arenas-Huertero C, Chua NH. 2012. Genome-wide analysis uncovers regulation of long intergenic noncoding RNAs in Arabidopsis. Plant Cell 24(11): 43334345.

Liu X, Sun H, Chen W, Guo Q, Xiaolin LI, Liang G. 2016. Advances in studies on carotenoids in loquat fruit. Journal of Fruit Science 35(6): 932-939.

Liu Z, Zhang Y, Liu R, Hao H, Wang Z, Bi Y. 2011. Phytochrome interacting factors (PIFs) are essential regulators for sucrose-induced hypocotyl elongation in Arabidopsis. Journal of Plant Physiology 168(15): 1771-1779.

Lobo I. 2012. Basic local alignment search tool (BLAST). J Mol Biol 215(3): 403-10.

Lockhart J. 2014. How ELONGATED HYPOCOTYL5 helps protect plants from UV-B rays. The Plant Cell 26(10): 3826-3826. 
778

779

780

781

782

783

784

785

786

787

788

789

790

791

792

793

794

795

796

797

798

Lorrain S, Allen T, Duek PD, Whitelam GC, Fankhauser C. 2008. Phytochrome-mediated inhibition of shade avoidance involves degradation of growth-promoting bHLH transcription factors. Plant Journal 53(2): 312-323.

Maier A, Hoecker U. 2015. COP1/SPA ubiquitin ligase complexes repress anthocyanin accumulation under low light and high light conditions. Plant Signaling \& Behavior 10(1): e970440.

Moeder W, Phan V, Yoshioka K. 2019. $\mathrm{Ca}^{2+}$ to the rescue $-\mathrm{Ca}^{2+}$ channels and signaling in plant immunity. Plant Science 279: 19.

Mugford SG, Lee BR, Koprivova A, Matthewman C, Kopriva S. 2011. Control of sulfur partitioning between primary and secondary metabolism. Plant Journal 65(1): 96-105.

Nafie EM. 2015. Effect of brassinolide application on growth certain metabolic activities and yield of tomato. Egyptian Journal of Physiological Sciences 1:103-117.

Oh E, Zhu JY, Wang ZY. 2012. Interaction between BZR1 and PIF4 integrates brassinosteroid and environmental responses. Nature Cell Biology 14(8): 802-809.

Ouyang X, Li J, Li G, Li B, Chen B, Shen H, Huang X, Mo X, Wan X, Lin R, Li S, Wang H, Deng XW. 2011. Genome-wide binding site analysis of FAR-RED ELONGATED HYPOCOTYL3 reveals its novel function in Arabidopsis development. Plant Cell 23(7): 2514-2535.

Pacín M, Legris M, Casal JJ. 2013. COP1 re-accumulates in the nucleus under shade. Plant Journal for Cell \& Molecular Biology 75(4): 631-641.

Pierik R, Djakovic-Petrovic T, Keuskamp DH, de Wit M, Voesenek LACJ. 2009. Auxin and ethylene regulate elongation responses to neighbor proximity signals independent of gibberellin and DELLA proteins in Arabidopsis. Plant Physiology 149(4): 1701-1712. 
799

800

801

802

803

804

805

806

807

808

809

810

811

812

813

814

815

816

817

818

819

Qin Z, Tan X, Ning H, Jing HU, Miao Y, Zhang X. 2018. Quantification and characterization of polysaccharides from different aged Dendrobium officinale stems. Food Science 39(6): 189-193.

Qiu YY, Tang LQ, Wei W. 2017. Berberine exerts renoprotective effects by regulating the AGEs-RAGE signaling pathway in mesangial cells during diabetic nephropathy[J]. Molecular and Cellular Endocrinology 443:89-105.

Rausenberger J, Tscheuschler A, Nordmeier W, Wüst F, Timmer J, Schifer E, Fleck C, Hiltbrunner A. 2011. Photoconversion and nuclear rrafficking cycles determine phytochrome $A \backslash$ "s response profile to far-red light. Cell 146(5): 0-825.

Robson F, Okamoto H, Patrick E, Harris SR, Wasternack C, Brearley C, Turner JG. 2010. Jasmonate and phytochrome A signaling in Arabidopsis wound and shade responses are integrated through JAZ1 stability. Plant Cell 22(4): 1143-1160.

Rodrigo L, Daniela H, Abraham M, Jan WDC, Janine H, Erika C, Alessandra A, Akifumi A, Tobias Z, Felipe A, Simone DC. 2016. The photomorphogenic factors UV-B RECEPTOR 1, ELONGATED HYPOCOTYL 5, and HY5 HOMOLOGUE are part of the UV-B signalling pathway in grapevine and mediate flavonol accumulation in response to the environment. Journal of Experimental Botany 67(18): 5429-5445.

Sara K, Geonhee H, Seulgi L, Zhu JY, Inyup P, Thi NT, Jungmook K, Eunkyoo O. 2017. High ambient temperature represses anthocyanin biosynthesis through degradation of HY5. Frontiers in Plant Science 8: 1787-1797.

Schambow TJ, Adjesiwor AT, Lorent L, Kniss AR. 2019. Shade avoidance cues reduce Beta vulgaris growth. Weed Science 67(3): 1-7. 
820

821

822

823

824

825

826

827

828

829

830

831

832

Sellaro R, Yanovsky MJ, Casal JJ. 2011. Repression of shade-avoidance reactions by sunfleck induction of HY5 expression in Arabidopsis. Plant Journal for Cell \& Molecular Biology 68(5): 919-928.

Shan CJ, Zhang SL, Li DF, Zhao YZ, Tian XL, Zhao XL, Wu Y, Wei X, Liu R. 2011. Effects of exogenous hydrogen sulfide on the ascorbate and glutathione metabolism in wheat seedlings leaves under water stress. Acta Physiologiae Plantarum 33(6): 2533-2540.

Sharkhuu A, Narasimhan ML, Merzaban JS, Bressan RA, Weller S, Gehring C. 2014. A red and far-red light receptor mutation confers resistance to the herbicide glyphosate. The Plant Journal 78(6): 916-926.

Shen JG, Yao MF, Chen XC, Feng YF, Ye YH, Tong ZH. 2009. Effects of puerarin on receptor for advanced glycation end products in nephridial tissue of streptozotocin-induced diabetic rats[J]. Molecular Biology Reports 36(8):2229-2233.

Shi Q, Zhang H, Song X, Jiang YE, Li G. 2018. Functional characterization of the maize phytochromeinteracting factors PIF4 and PIF5. Front Plant Sci 8: 2273-2285.

Shin DH, Choi M, Kim K, Bang G, Cho M, Choi SB, Choi G. 2013. Park YI, HY5 regulates anthocyanin biosynthesis by inducing the transcriptional activation of the MYB75/PAP1 transcription factor in Arabidopsis. Febs Letters 587(10): 1543-1547.

Shuai P, Liang D, Tang S, Zhang Z, Ye CY, Su Y, Xia X, Yin W. 2014. Genome-wide identification and functional prediction of novel and drought-responsive lincRNAs in Populus trichocarpa. Journal of Experimental Botany 65(17):4975-83.

Sun H, Qiang HU, Jin H, Wang YZ. 2017. Research advances in chemical constituents and pharmacological activities of Dendrobii Officinalis caulis. Chinese Journal of Experimental Traditional Medical Formulae 23(11): 225-234. 
841

842

843

844

845

846

847

848

849

850

851

852

853

854

855

856

857

858

859

860

861

Sun XL, Yue-Fei XU, Lu-Yi MA. 2010. A review of acclimation of photosynthetic pigment composition in plant leaves to shade environment. Chinese Journal of Plant Ecology 8: 989-999.

Vanyushin BF, Ashapkin VV. 2011. DNA methylation in higher plants: Past, present and future. Biochim Biophys Acta 1809(8): 0-368.

Wang H, Chung PJ, Liu J, Jang IC, Chua NH. 2014. Genome-wide identification of long noncoding natural antisense transcripts and their responses to light in Arabidopsis. Genome Research 24(3): 444-453.

Wang H, Feng Q, Zhang M, Yang C, Sha W, Liu B. 2010. Alteration of DNA methylation level and pattern in sorghum (Sorghum bicolor L.) pure-lines and inter-line F1 hybrids following low-dose laser irradiation. Journal of Photochemistry \& Photobiology B Biology 99(3): 150-153.

Wang J, Meng X, Dobrovolskaya OB, Orlov YL, Chen M. 2017. Non-coding RNAs and their roles in stress response in plants. Genomics, Proteomics \& Bioinformatics 15(5), 301-312.

Wang KK, Chen SQ, Wang W, Xie Q, Chen QX. 2016. Antimicrobial activity of extracts from Dendrobium officinale Kimura et Migo grown under four cultivation methods. Fujian Journal of Agricultural Sciences 3: $236-241$.

Wang WT, Zhang JW, Wang D, Tao SH, Ji YL, Wu B. 2010. Relation between light qualities and accumulation of steroidal glycoalkaloids as well as signal molecule in cell in potato tubers. Acta Agronomica Sinica 36(4): 629-635.

Warpeha KM, Montgomery BL. 2015. Light and hormone interactions in the seed-to-seedling transition. Environmental \& Experimental Botany 83: 56-65.

Won C, Shen X, Mashiguchi K, Zheng Z, Dai X, Cheng Y, Kasahara H, Kamiya Y, Chory J, Zhao Y. 2011. Conversion of tryptophan to indole-3-acetic acid by TRYPTOPHAN AMINOTRANSFERASES 
862

863

864

865

866

867

868

869

870

871

872

873

874

875

876

878

879

880

881

882

OF ARABIDOPSIS and YUCCAs in Arabidopsis. Proceedings of the National Academy of Sciences of the United States of America 108(45): 18518-18523.

Xie Y, Liu Y, Wang H, Ma X, Wang B, Wu G, Wang H. 2017. Phytochrome-interacting factors directly suppress MIR156 expression to enhance shade-avoidance syndrome in Arabidopsis. Nature Communications 8(1): 348-358.

Xie Y, Zhang W, Duan X, Dai C, Zhang YH, Cui W, Wang R, Shen W. 2015. Hydrogen-rich wateralleviated ultraviolet-B-triggered oxidative damage is partially associated with the manipulation of the metabolism of (iso)flavonoids and antioxidant defence in Medicago sativa. Functional Plant Biology 42(12): 1141-1157.

Xu BQ, Cui YY, Guo C. 2012. Dynamic variation of biomass and content of polysaccharide and alkaloid in protocorm like bodies from Dendrobium officinale at different light intensities and incubation time. Chinese Traditional \& Herbal Drugs 43(2): 355-359.

Yamashita O, Katayama S, Senga Y, Sugiyama Y, Sueyoshi N, Kameshita I. 2018. Characterization of CoPK02, a $\mathrm{Ca}^{2+} /$ calmodulin-dependent protein kinase in mushroom Coprinopsis cinerea. Bioence Biotechnology and Biochemistry 82(8): 1-9.

Yang C, Xie F, Jiang Y, Li Z, Li L. 2017. Phytochrome A negatively regulates the shade avoidance response by increasing Auxin/Indole acidic acid protein stability. Developmental Cell 44(1): 29-41.

Yang CM, Yang ZX, Ma XL. 2019. The Mechanism of AGEs-RAGE Signaling Pathway in Diabetic Nephropathy and the Progress of Chinese Medicine Treatment[J]. Acta Chinese Medicine 9(34):18641868.

Yi T, Ferrer JL, Ljung K, Pojer F, Hong F, Long JA, Moreno JE, Bowman ME, Ivans LJ, Cheng Y, 
883

884

885

886

887

888

889

890

891

892

893

894

895

896

897

898

899

900

901

902

903

Lim J, Zhao Y, Ballaré CL, Sandberg G, Noel JP, Chory J. 2008. Rapid synthesis of auxin via a new tryptophan-dependent pathway is required for shade avoidance in plants. Cell 133(1): 0-176.

Yin DK, Yang Y, Chen S, Li Y, Gao XD. 2012. Effects of polysaccharides from Coptis Chinensis on HUVECs proliferation induced by advanced glycation endproducts and expression of its receptor[J]. Pharmaceutical Biotechnology 19(6):476-479.

Yu G, Wang L G, Han Y, He Q. 2012. clusterProfiler: an R package for comparing biological themes among gene clusters. Omics: a journal of integrative biology 16(5): 284-287.

Yuan Y, Hou XF, Feng L, Jia XB. 2017. Inhibition of puerarin on formation of advanced glycation end products in vivo and in vitro[J]. Chinese Traditional and Herbal Drugs 48(7):1386-1390.

Zhang GQ, Xu Q, Bian C, Tsai WC, Liu ZJ. 2016. The Dendrobium catenatum Lindl. genome sequence provides insights into polysaccharide synthase, floral development and adaptive evolution. Scientific Reports 6(19029): 1-10.

Zheng X, Wu S, Zhai H, Zhou P, Yang J. 2013. Arabidopsis phytochrome B promotes SPA1 nuclear accumulation to repress photomorphogenesis under far-red light. Plant Cell 25(1): 115-133.

Zhou F. 2012. Advances in domain structure and photochemistry of plant photoreceptors. Guangdong Agricultural Sciences 10: 234-236.

Zhou P, Song M, Yang Q, Su L, Hou P, Guo L, Zheng X, Xi Y, Meng F, Xiao Y, Yang L, Yang J. 2014. Both PHYTOCHROME RAPIDLY REGULATED1 (PAR1) and PAR2 promote seedling photomorphogenesis in multiple light signaling pathways. Plant Physiology 164(2): 841-852.

Zhu D, Maier A, Lee JH, Laubinger S, Saijo Y, Wang H, Qu LJ, Hoecker U, Deng XW. 2008. Biochemical characterization of Arabidopsis complexes containing CONSTITUTIVELY 
PHOTOMORPHOGENIC1 and SUPPRESSOR OF PHYA proteins in light control of plant development.

905

Plant Cell 20(9): 2307-2323. 


\section{Table $\mathbf{1}$ (on next page)}

Table 1 Phenotype of $D$. officinale after different light treatments 
1

\begin{tabular}{|c|c|c|c|c|c|c|c|c|c|}
\hline \multirow[b]{2}{*}{ Treatment } & \multicolumn{3}{|c|}{ CK } & \multicolumn{3}{|c|}{ FR1 } & \multicolumn{3}{|c|}{ FR4 } \\
\hline & $\begin{array}{c}\text { Average } \\
\text { value }\end{array}$ & $\begin{array}{l}\text { Standard } \\
\text { deviation }\end{array}$ & $\begin{array}{c}\text { Duncan } \\
(5 \%)\end{array}$ & $\begin{array}{c}\text { Average } \\
\text { value }\end{array}$ & $\begin{array}{l}\text { Standard } \\
\text { deviation }\end{array}$ & $\begin{array}{c}\text { Duncan } \\
(5 \%)\end{array}$ & $\begin{array}{c}\text { Average } \\
\text { value }\end{array}$ & $\begin{array}{l}\text { Standard } \\
\text { deviation }\end{array}$ & $\begin{array}{c}\text { Duncan } \\
(5 \%)\end{array}$ \\
\hline $\begin{array}{l}\text { Plant } \\
\text { height } \\
(\mathrm{mm})\end{array}$ & 50.90 & 0.64 & $\mathrm{c}$ & 58.20 & 0.87 & b & 68.22 & 0.64 & a \\
\hline $\begin{array}{c}\text { Stem } \\
\text { diameter } \\
(\mathbf{m m})\end{array}$ & 4.45 & 0.07 & b & 4.62 & 0.05 & $\mathrm{a}$ & 4.64 & 0.04 & $\mathrm{a}$ \\
\hline $\begin{array}{c}\text { Leaf area } \\
\qquad\left(\mathbf{m m}^{2}\right)\end{array}$ & 93.34 & 1.09 & $\mathrm{c}$ & 105.00 & 1.24 & $\mathrm{~b}$ & 125.37 & 1.19 & $\mathrm{a}$ \\
\hline $\begin{array}{l}\text { Number } \\
\text { of leaves }\end{array}$ & 5 & - & - & 5 & - & - & 5 & - & - \\
\hline
\end{tabular}

2 
Table 2 (on next page)

Table 2 mRNA and IncRNA results from nine $D$. officinale libraries 
1

\begin{tabular}{ccccccc}
\hline Samples & Total reads & Total mapped reads (\%) & $\begin{array}{c}\text { Uniquely mapped reads } \\
\mathbf{( \% )}\end{array}$ & $\begin{array}{c}\text { Q20 } \\
\text { (\%) }\end{array}$ & $\begin{array}{c}\text { Q30 } \\
\text { (\%) }\end{array}$ & $\begin{array}{c}\text { GC content } \\
\text { (\%) }\end{array}$ \\
\hline CK1 & $88,743,106$ & 85.91 & 78.90 & 98.73 & 95.69 & 42.48 \\
CK2 & $66,153,614$ & 86.69 & 80.01 & 98.85 & 96.01 & 43.11 \\
CK3 & $74,195,304$ & 86.19 & 78.87 & 98.87 & 95.95 & 42.46 \\
FR1-1 & $65,759,200$ & 86.51 & 79.94 & 98.71 & 95.79 & 42.66 \\
FR1-2 & $66,959,230$ & 85.48 & 79.32 & 98.61 & 95.49 & 42.52 \\
FR1-3 & $77,913,256$ & 85.47 & 78.40 & 98.53 & 95.47 & 43.02 \\
FR4-1 & $66,569,014$ & 85.03 & 79.12 & 98.78 & 95.76 & 43.16 \\
FR4-2 & $67,769,478$ & 85.26 & 79.58 & 98.74 & 95.66 & 43.00 \\
FR4-3 & $70,015,212$ & 84.16 & 79.01 & 98.66 & 95.51 & 42.65 \\
\hline
\end{tabular}

2

3 


\section{Table 3 (on next page)}

Table 3 Top five GO terms obtained from an enrichment analysis of the target genes of DE InCRNAs in $D$. officinale under different light treatments 


\begin{tabular}{|c|c|c|c|c|c|c|}
\hline & \multirow{2}{*}{ ID } & \multirow{2}{*}{ Description } & \multicolumn{3}{|c|}{ q value } & \multirow{2}{*}{ geneID } \\
\hline & & & FR1- & FR4- & FR4- & \\
\hline \multirow{19}{*}{$\begin{array}{c}\text { Biological } \\
\text { process }\end{array}$} & GO:001035 & leaf shaping & 0.01280 & & 0.02798 & gene-MA16_Dca015315;gene-MA16_Dca018761 \\
\hline & GO:000046 & maturation of $5.8 \mathrm{~S}$ rRNA & 0.01280 & & & gene-MA16_Dca018926;gene-MA16_Dca018927 \\
\hline & GO:000974 & response to brassinosteroid & 0.01280 & & 0.03869 & gene-MA16_Dca015315;gene-MA16_Dca018761 \\
\hline & GO: 190100 & ubiquinone- 6 biosynthetic process & 0.01280 & 0.07223 & & gene-MA16_Dca014146;gene-MA16_Dca014147 \\
\hline & GO:190262 & assembly of large subunit precursor of & 0.01280 & & & gene-MA16_Dca018926;gene-MA16_Dca018927 \\
\hline & GO:009750 & sialylation & & 0.07223 & & gene-MA16_Dca009824;gene-MA16_Dca013452 \\
\hline & GO:000598 & sucrose biosynthetic process & & 0.08464 & & gene-MA16_Dca009826 \\
\hline & GO:000629 & nucleotide-excision repair, DNA incision, & & 0.08464 & & gene-MA16_Dca007683 \\
\hline & GO:000642 & isoleucyl-tRNA aminoacylation & & 0.08464 & & gene-MA16_Dca009286 \\
\hline & GO:001631 & & & & & gene-MA16_Dca000617;gene-MA16_Dca013817;gene- \\
\hline & 1 & dephosphorylation & & & 0.03869 & MA16_Dca015948; \\
\hline & GO:007058 & calcium ion transmembrane transport & & & 0.03869 & gene-MA16_Dca015955;gene-MA16_Dca022336;gene- \\
\hline & GO:001613 & brassinosteroid biosynthetic process & & & 0.03869 & gene-MA16_Dca015315;gene-MA16_Dca018760;gene- \\
\hline & GO:000578 & signal recognition particle, endoplasmic & 010451 & & & gene-MA16 Dca000647.gene-MA16 Dca000982 \\
\hline & 6 & reticulum targeting & 0.10401 & & & 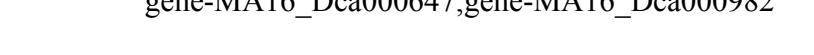 \\
\hline & GO:007065 & HAUS complex & 0.10451 & & & gene-MA16_Dca017504;gene-MA16_Dca026687 \\
\hline & GO:000579 & Golgi medial cisterna & 0.16459 & & & MA16_Dca001958 \\
\hline & $\begin{array}{c}\text { GO: } 000585 \\
2\end{array}$ & $\begin{array}{l}\text { eukaryotic translation initiation factor } 3 \\
\text { complex }\end{array}$ & 0.16459 & & & gene-MA16_Dca001383;gene-MA16_Dca001386 \\
\hline & GO:000587 & kinesin complex & 0.16459 & & & gene-MA16_Dca013578;gene-MA16_Dca026695 \\
\hline
\end{tabular}


GO:001602 membrane

0

GO:000011 nucleotide-excision repair factor 1

GO:000015 cytoplasmic ubiquitin ligase complex

GO:000567 transcription factor TFIIA complex

GO:000970 chloroplast inner membrane

GO:000580 cis-Golgi network

GO:000578 endoplasmic reticulum

GO:000579 Golgi medial cisterna

GO:000562 intracellular

GO:000374

translation initiation factor activity

0.00214

GO:001001 steroid 22-alpha hydroxylase activity $\quad 0.00543$

GO:004573 nutrient reservoir activity 0.00543

GO:003014 manganese ion binding
0.00913

\subsection{8}

0.19328

$0.19328 \quad 0.17955$

0.24481

0.09257

0.17955

0.17955

0.17955

gene-MA16_Dca000800;gene-MA16_Dca000921;gene-

MA16_Dca002727;

gene-MA16_Dca002728;gene-MA16_Dca003087;gene-

MA16_Dca005684;

gene-MA16_Dca006160;gene-MA16_Dca007647;gene-

MA16_Dca008165;

gene-MA16_Dca009282;gene-MA16_Dca009818;gene-

MA16_Dca012412;

gene-MA16_Dca013211;gene-MA16_Dca013212;gene-

MA16_Dca013412;

gene-MA16 Dca014148;gene-MA16 Dca014149;gene-

gene-MA16_Dca007683

gene-MA16_Dca006393

gene-MA16 Dca004588

gene-MA16_Dca015955;gene-MA16_Dca016038

gene-MA16_Dca000620;gene-MA16_Dca001958

gene-MA16_Dca003087;gene-MA16_Dca015315;gene-

MA16_Dca015955;

gene-MA16_Dca017315;gene-MA16_Dca018760;genegene-MA16_Dca001958

gene-MA16_Dca012408;gene-MA16_Dca012411;gene-

MA16_Dca013217;

gene-MA16_Dca015311;gene-MA16_Dca015854;genegene-MA16_Dca001383;gene-MA16_Dca001386;geneMA16_Dca002840;

gene-MA16_Dca018345;gene-MA16_Dca018926;gene-

gene-MA16_Dca015315;gene-MA16_Dca018761

gene-MA16_Dca023265;gene-MA16_Dca023266;gene-

gene-MA16 Dca023265;gene-MA16 Dca023266;gene- 


\begin{tabular}{|c|c|c|c|c|c|}
\hline GO:000395 & NADH dehydrogenase activity & 0.02937 & 0.06782 & & gene-MA16_Dca014146;gene-MA16_Dca014147 \\
\hline GO:000817 & O-methyltransferase activity & & 0.01400 & & $\begin{array}{c}\text { gene-MA16_Dca007905;gene-MA16_Dca007906;gene- } \\
\text { MA16_Dca007907; }\end{array}$ \\
\hline GO:000484 & ureidoglycolate hydrolase activity & & 0.01400 & & gene-MA16_Dca005252;gene-MA16_Dca005253 \\
\hline GO:000837 & sialyltransferase activity & & 0.05484 & & gene-MA16_Dca009824;gene-MA16_Dca013452 \\
\hline GO:000538 & calcium-transporting ATPase activity & & 0.12133 & 0.02220 & gene-MA16_Dca015955;gene-MA16_Dca021262 \\
\hline GO:001526 & channel activity & & & 0.00196 & gene-MA16_Dca017309;gene-MA16_Dca017310;gene- \\
\hline GO:001001 & steroid 22-alpha hydroxylase activity & & & 0.01496 & gene-MA16_Dca015315;gene-MA16_Dca018761 \\
\hline GO:0000386 & 3-oxo-5-alpha-steroid 4-dehydrogenase & & & 0.02220 & gene-MA16_Dca007792;gene-MA16_Dca007793 \\
\hline $\begin{array}{c}\text { GO:001916 } \\
6\end{array}$ & $\begin{array}{l}\text { trans-2-enoyl-CoA reductase (NADPH) } \\
\text { activity }\end{array}$ & & & 0.03513 & gene-MA16_Dca007792;gene-MA16_Dca007793 \\
\hline
\end{tabular}




\section{Figure 1}

Figure 1 Phenotype of $D$. officinale under different light treatments.

A-C: Phenotype of $D$. officinale before the different light treatments; D-E: Phenotype of $D$. officinale after the different light treatments. A and D, CK; B and E, FR1; C and F, FR4. Red light intensity:blue light intensity:far-red light intensity ratios: 100:100:0, CK; 80:80:40, FR1; and 40:40:120, FR4. Bars=10 mm. 

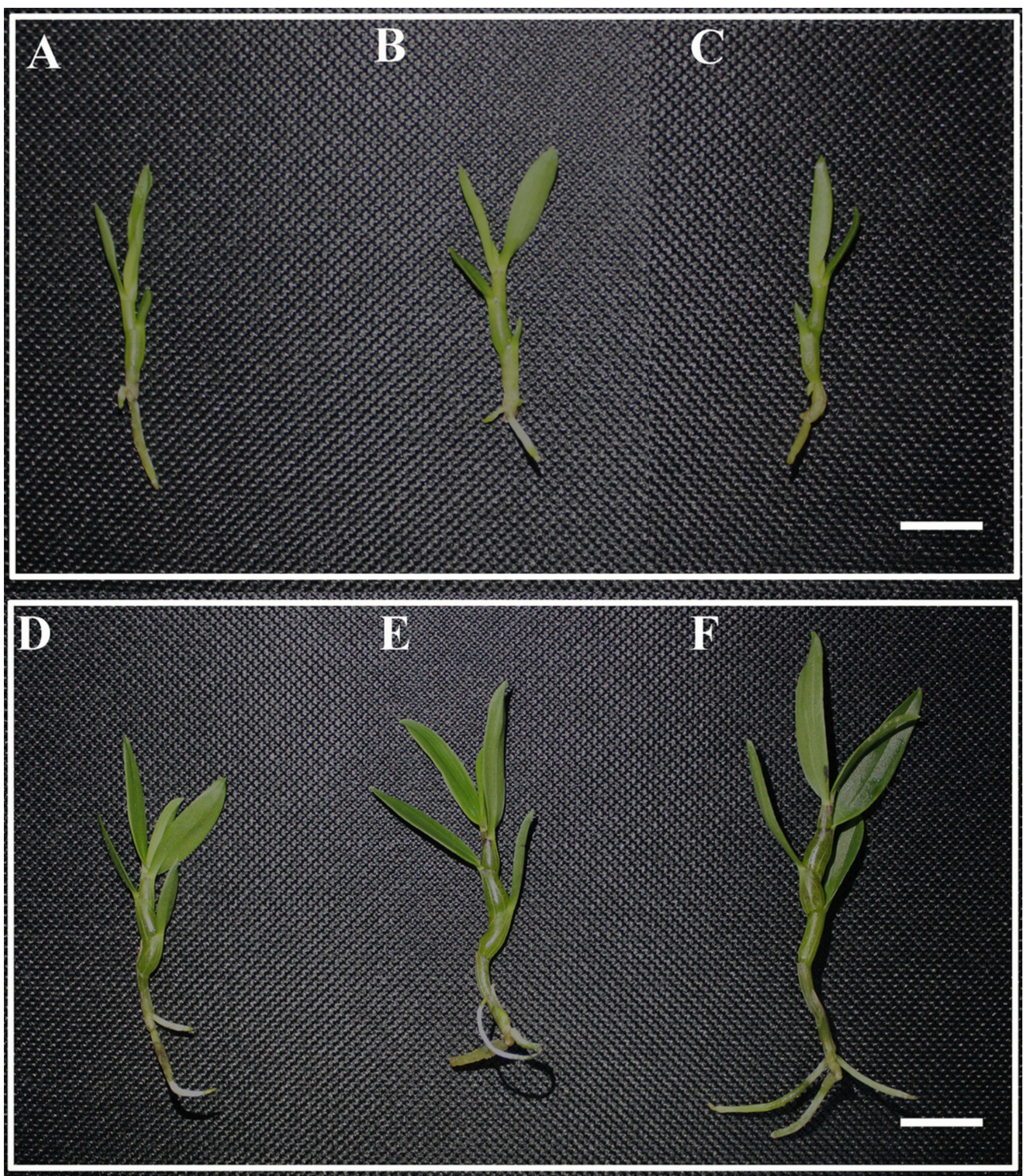
Figure 2

Figure 2 DE IncRNAs under the different light treatments.

A, Number of IncRNAs with high (FPKM $>50)$, moderate $(5 \leq$ FPKM $\leq 50)$ and low (FPKM $<5)$ expression in each library. B, Venn diagram showing the numbers of unique and commonly regulated IncRNAs identified from the FR1-CK and FR4-CK comparisons. C, DE IncRNAs in response to different light treatments. The scale from red to blue corresponds to the numerical value of $\log _{2}(\mathrm{FPKM})$ from high to low.
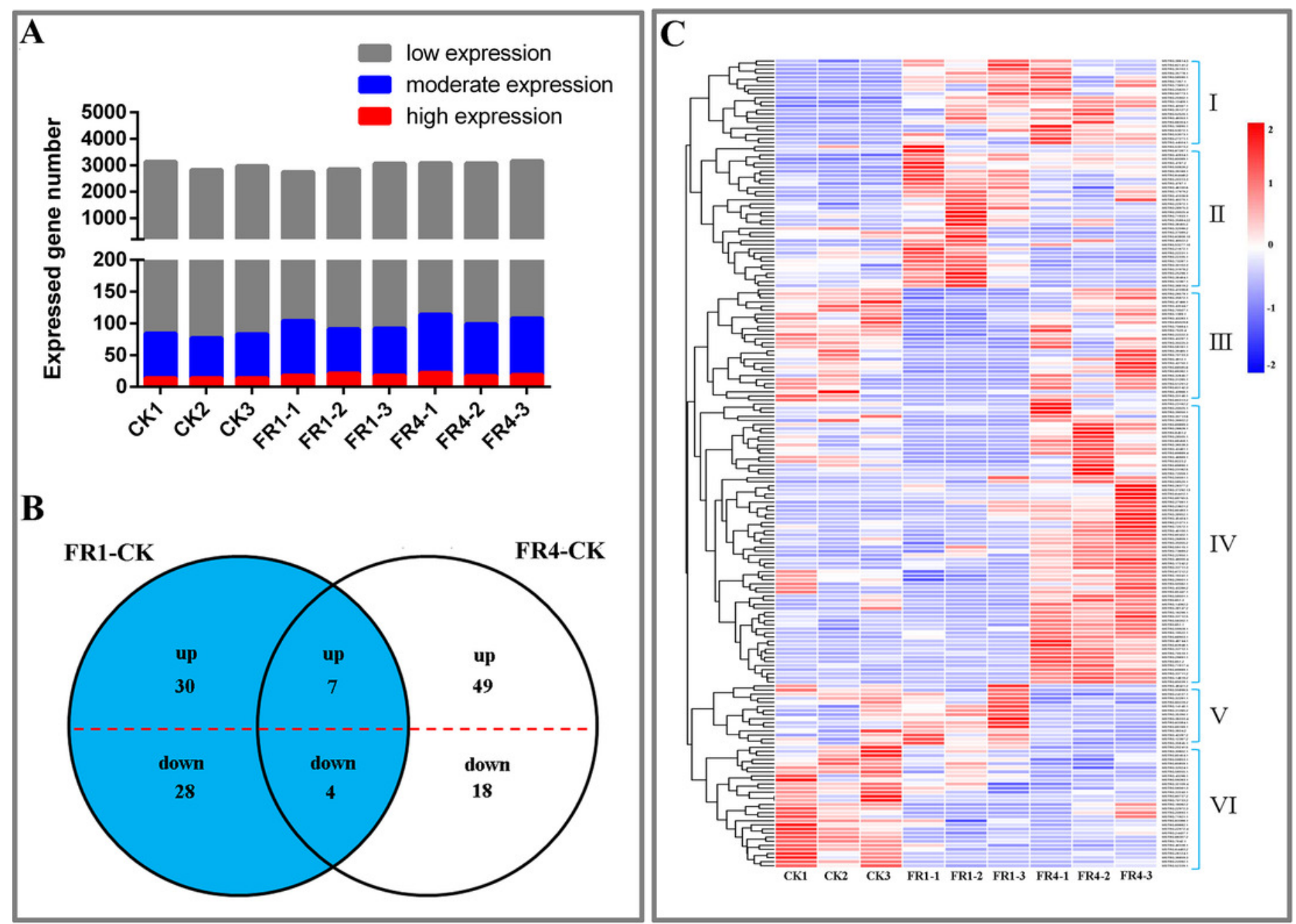
Figure 3

Figure 3 KEGG enrichment analysis of the target genes of DE IncRNAs in D. officinale under different light treatments.

A, FR1-CK. B, FR4-CK. C, FR4-FR1. D, Top 20 KEGG pathways enriched in target genes of DE IncRNAs in the three groups. The red colour indicates that the comparison contains the indicated pathway, and the blue colour indicates that the comparison does not contain the pathway.

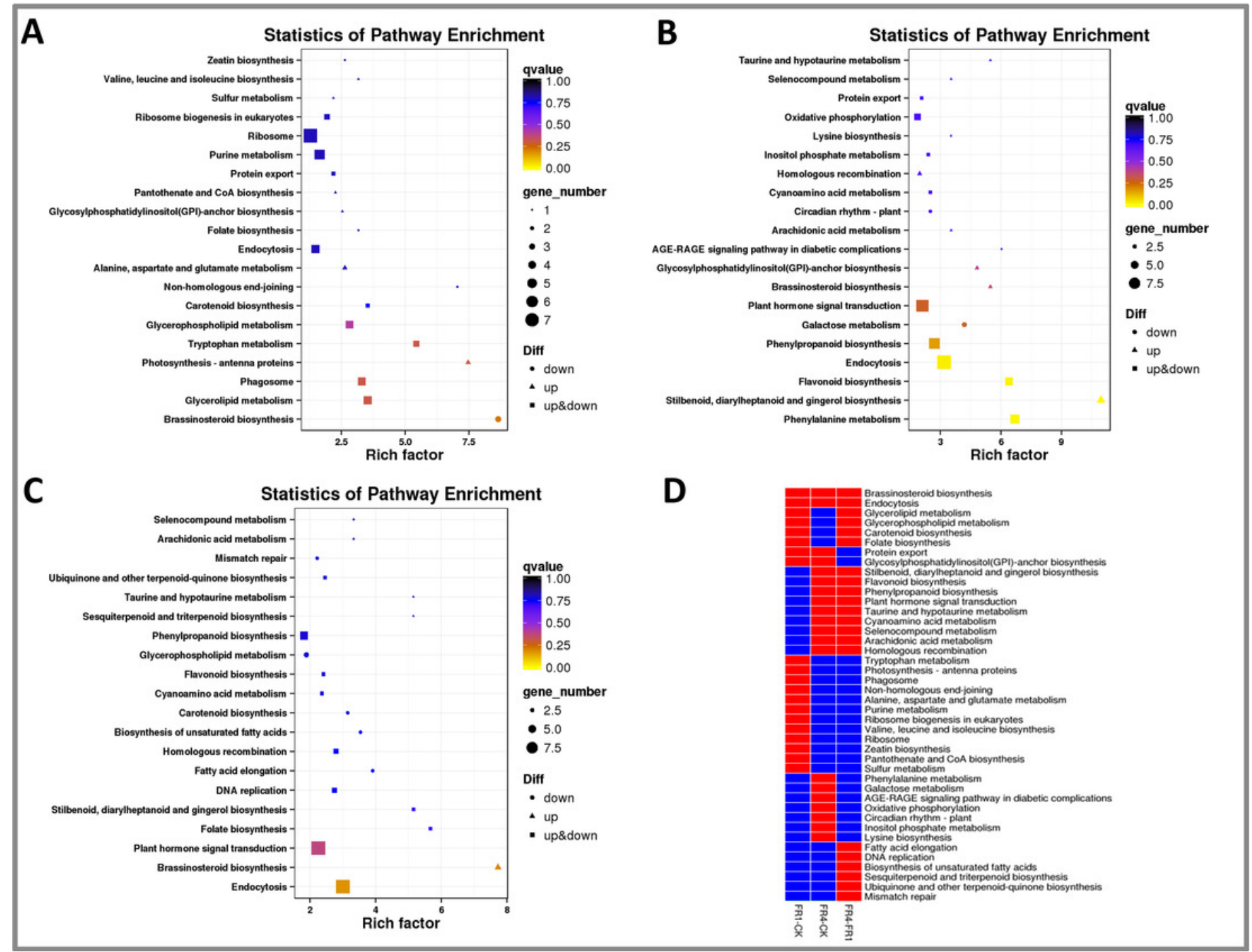




\section{Figure 4}

Figure 4 The relationship prediction of DE IncRNAs and their targets in $D$. officinale under different light treatments.

A, Interaction network of the results from the FR1-CK comparison. B, Interaction network of the results from the FR4-CK. The gradient-coloured circle nodes represent IncRNAs, and the blue circular nodes represent mRNAs. High expression and low expression of IncRNAs is shown in dark red and dark green respectively. The size of the circle indicates the number of connected genes. The solid lines indicate interaction associations between IncRNAs and mRNAs. 

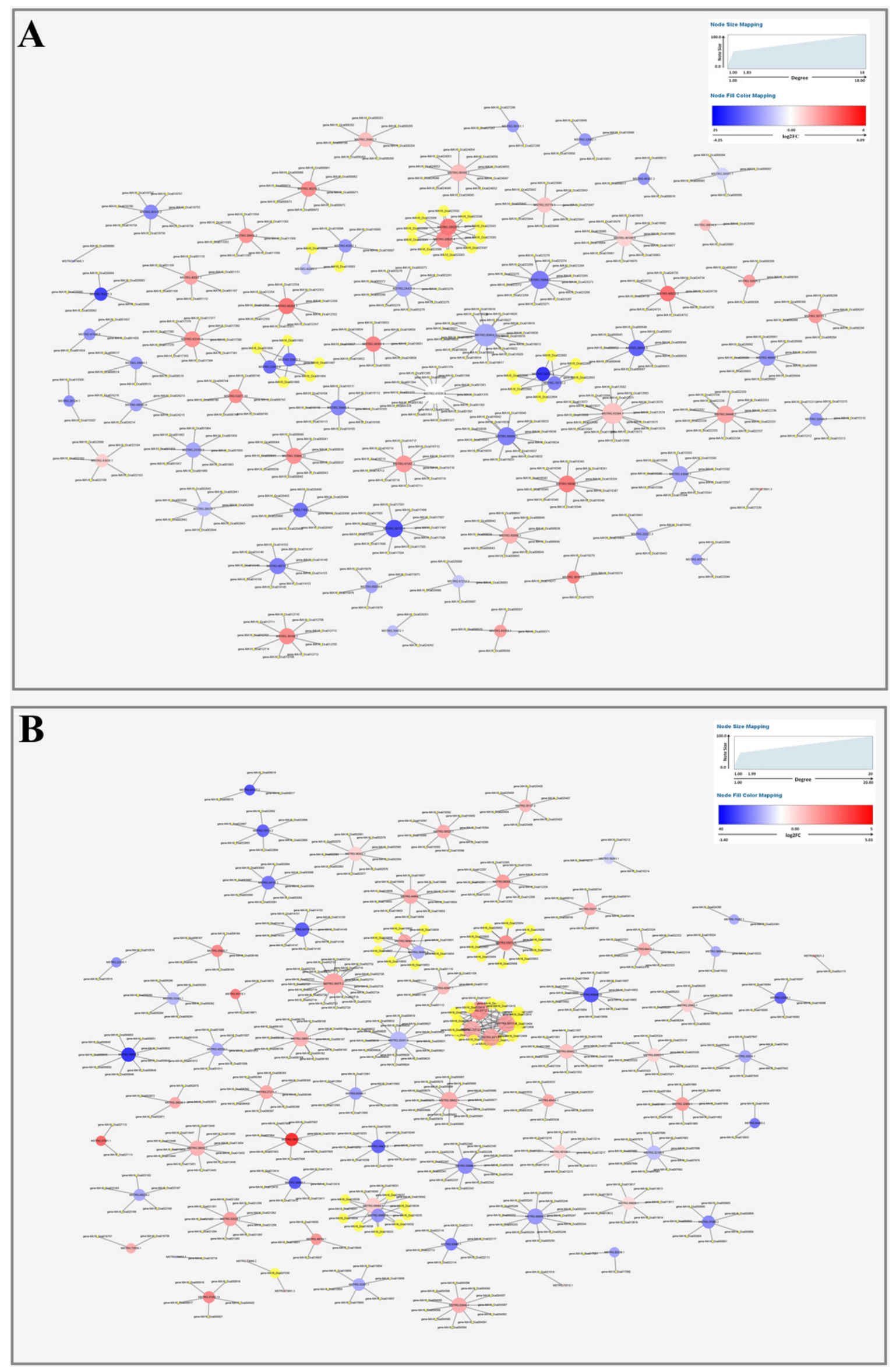


\section{Figure 5}

Figure 5 Metabolite contents in $D$. officinale under different light treatments.

A and B represent changes in the flavonoid contents in the leaves and stems respectively. C and $D$ represent changes in the alkaloid contents in the leaves and stems respectively. $E$ and $\mathrm{F}$ represent changes in the carotenoid contents in the leaves and stems respectively. $\mathrm{G}$ and $\mathrm{H}$ represent changes in the polysaccharide contents in the leaves and stems respectively. Different upper/lowercase letters indicate statistically significant differences at the 0.01/0.05 level, as determined by one-way ANOVA and Duncan's test. 

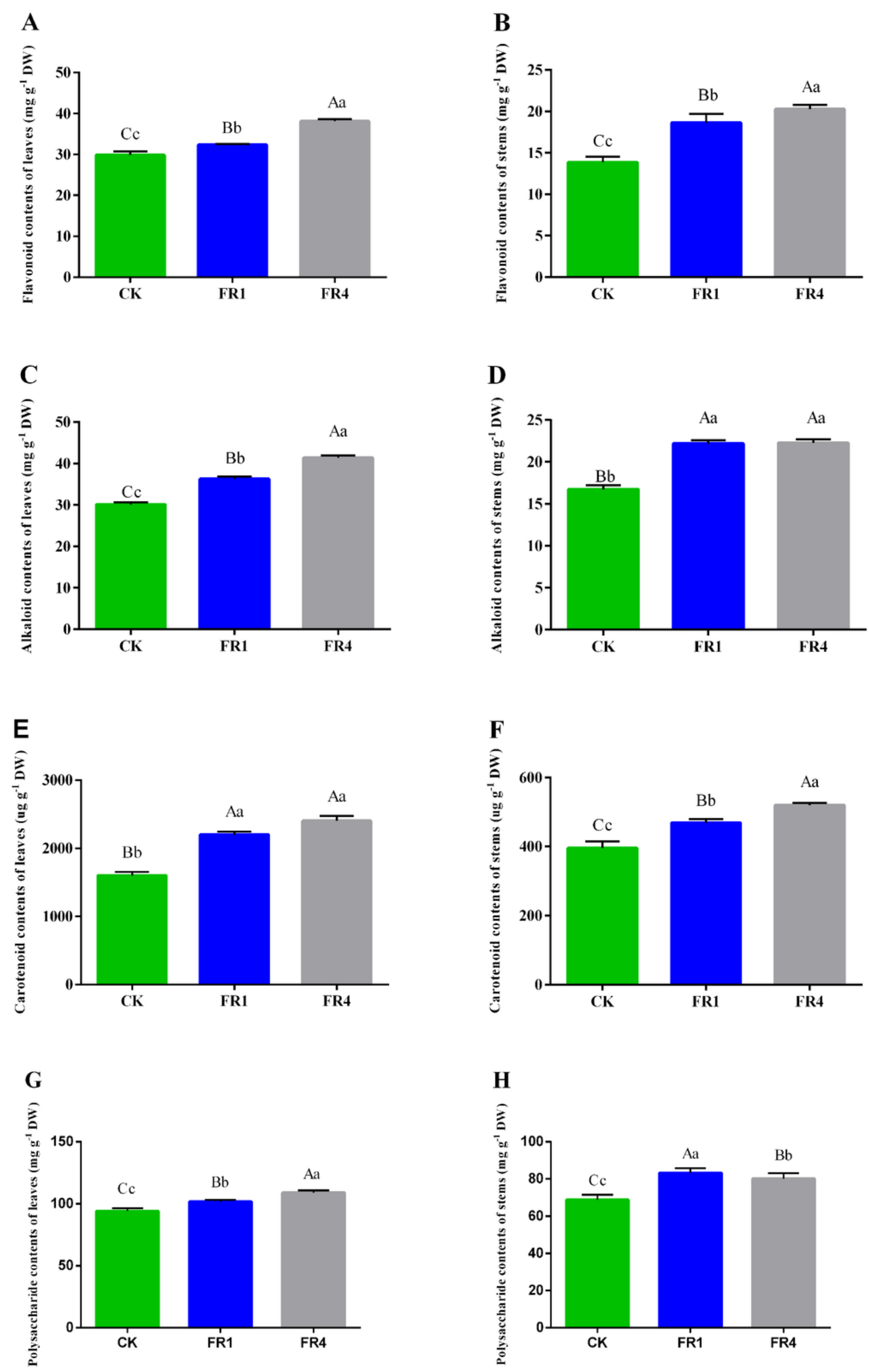
Figure 6

Figure 6 Levels of physiological and biochemical indicators in the leaves of $D$. officinale under different light treatments.

A, SOD activity; B, Relative membrane permeability; C, CaM content.

A

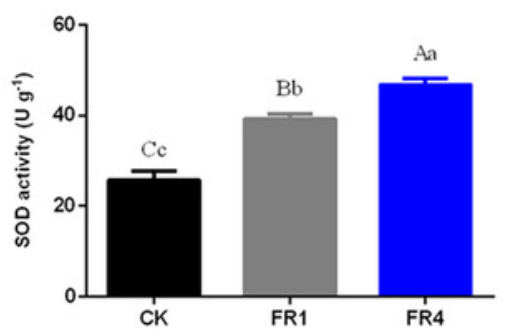

B

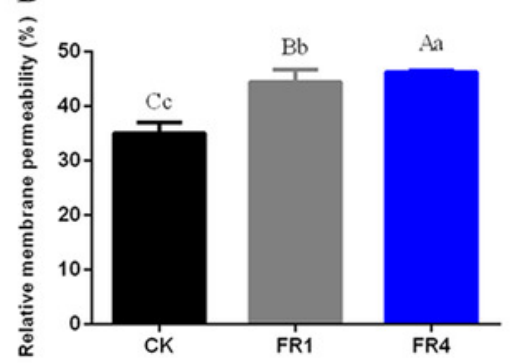

C

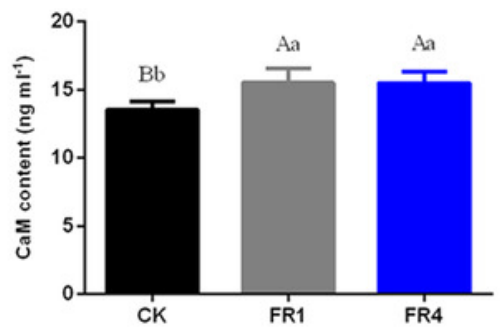




\section{Figure 7}

Figure 7 Identification of DE IncRNAs and their target genes in $D$. officinale under different far-red light conditions via qPCR.

$T A A 1$, tryptophan aminotransferase 1; CCD4, carotenoid cleavage dioxygenase; ZSD1, zerumbone synthase; GGT1_5, gamma-glutamyl transpeptidase 3-like; CYP90A1, cytochrome P450 family 90 subfamily A polypeptide 1 ; IAA, auxin-responsive protein IAA; ARR-A, twocomponent response regulator ARR-A family; PHYA, phytochrome A; COP1, constitutively photomorphogenic $1 ; H Y 5$, elongated hypocotyl 5; PIF3, phytochrome-interacting factor 3. 


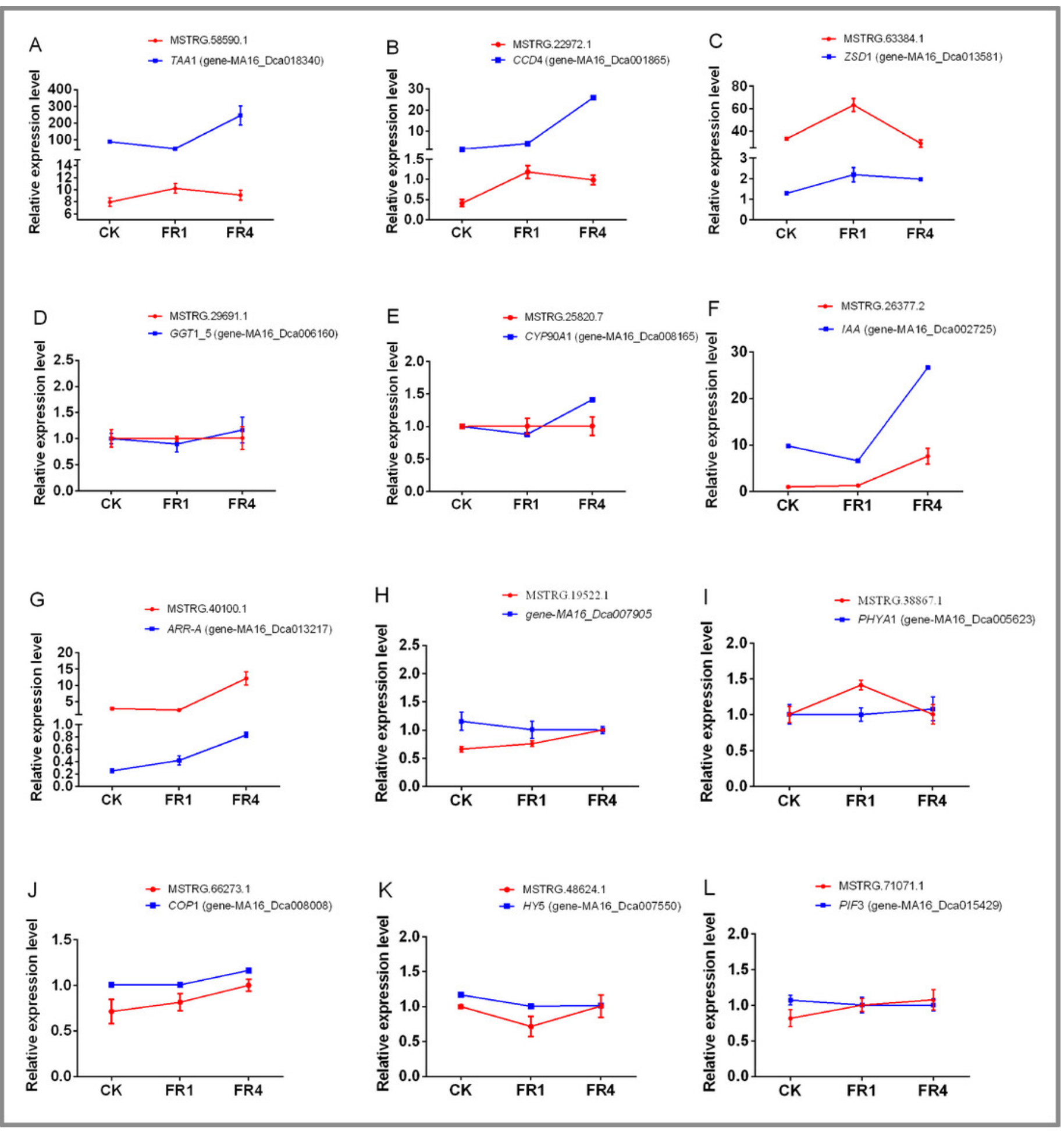


Figure 8

Figure 8 Model depicting the regulatory IncRNA-mediated mechanisms of photomorphogenesis under far-red light.

$\mathrm{FR}$, far-red light. The question mark indicates an uncertain relationship that requires further verification. 


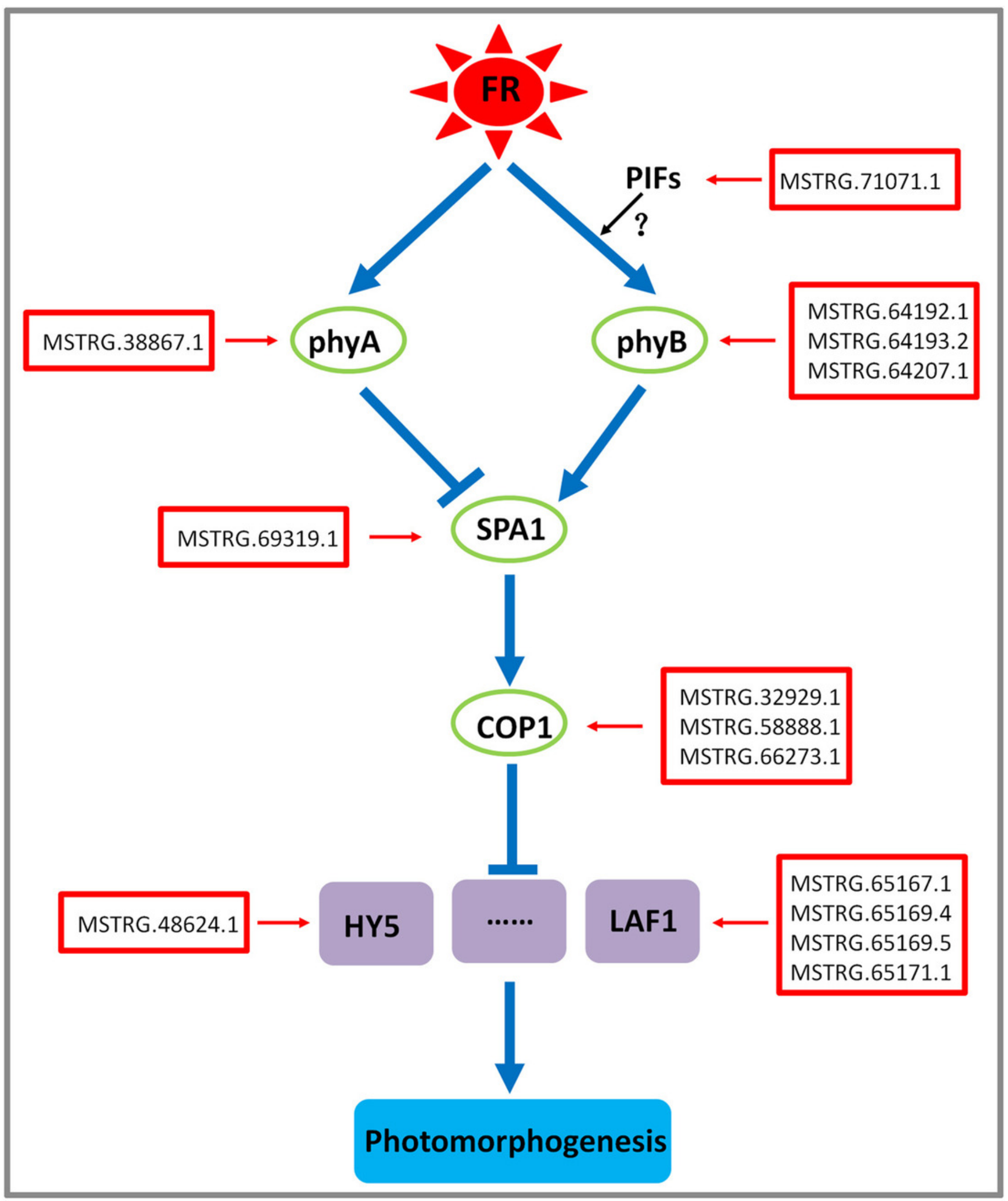

